\title{
Potential of Graphene-Polymer Composites for Ligament and Tendon Repair: A Review
}

\author{
Tânia Peixoto, Maria Conceição Paiva, António T. Marques, and Maria A. Lopes*
}

Tendon/ligament injuries are debilitating conditions that affect the life quality of a great percentage of the adult population. Several challenges still have to be addressed regarding the repair of these tissues, as current treatments show limited success. The use of biocompatible and biodegradable polymeric scaffolds potentially helps accomplish a complete and long-term functional repair but, unfortunately, these materials lack adequate mechanical properties to be used in such demanding applications. Graphene is a subject of interest for tissue-repair applications due to its electrical conductivity and mechanical properties. If incorporated adequately, it may significantly improve the physical properties of the composite, even at small loadings. Furthermore, graphene presents a biocompatible surface that may enhance cell adhesion, proliferation and differentiation and demonstrates promising outcomes in several in vitro and in vivo biological applications. Therefore, herein, the potential of graphene materials for the reinforcement of biodegradable polymers of interest for tendon/ligament repair is explored. The effect of graphene on relevant features such as mechanical properties, biodegradability, and biocompatibility is revised, to understand the feasibility of these composites to fulfill the requirements associated with these tissues and conclude how their applicability is extended to this field.

\section{Introduction}

Tendon and ligament injuries are one of the most prevailing health problems affecting the adult population worldwide. From the 30 million musculoskeletal injuries reported every

\author{
T. Peixoto, Prof. M. A. Lopes \\ REQUIMTE-LAQV \\ Departamento de Engenharia Metalúrgica e Materiais \\ Faculdade de Engenharia \\ Universidade do Porto \\ Rua Dr. Roberto Frias, 4200-465 Porto, Portugal \\ E-mail: malopes@fe.up.pt \\ T. Peixoto, Prof. M. C. Paiva \\ Instituto de Polímeros e Compósitos \\ Departamento de Engenharia de Polímeros \\ Universidade do Minho \\ 4800-058 Guimarães, Portugal \\ Prof. A. T. Marques \\ Departamento de Engenharia Mecânica \\ Faculdade de Engenharia \\ Universidade do Porto \\ Rua Dr. Roberto Frias, 4200-465 Porto, Portugal
}

The ORCID identification number(s) for the author(s) of this article can be found under https://doi.org/10.1002/adem.202000492.

DOI: 10.1002/adem.202000492 year, over half are thought to involve these tissues. ${ }^{[1-3]}$ These injuries not only can result in long-term pain, impaired function and disability, and so on, in reduced quality of life, but also represent significant economic costs: only in the United States (US), $\approx 30$ billion dollars are spent on musculoskeletal injuries each year, and only tendon/ligament injuries alone represent about $45 \%$ of these expenses. ${ }^{[1,3,4]}$ Furthermore, these injuries present a significant clinical challenge as although spontaneous healing can occur, it is a very slow process that frequently results in the formation of a scar tissue with low functionality. ${ }^{[2,3,5,6]}$

Due to this limited capability for selfhealing and the need for injured people to regain their functionality in a timely manner, surgical intervention is often required. ${ }^{[2,7]}$ Primary repairs involve the use of sutures to reattach the injured ends. However, this procedure is often ineffective, and it is not viable in case of extensive damage. In such cases, autografts or allografts have been used to reconstruct the lost tissue, but these present several disadvantages. ${ }^{[2,3,5,8,9]}$ As a result of this market demand, a variety of scaffolds have also been developed, some to share loads with the injured tissue or with the used biological graft and others to immediately replace the lost tissue and even promote new tissue formation. However, the development of a device that adequately mimics the mechanical performance of the native tissue remains challenging. ${ }^{[2,4,7,9-12]}$

In an attempt to meet the demanding mechanical requirements of these tissues, most devices have been developed using nondegradable polymers, such as poly(ethylene terephthalate) (PET), due to their high tensile properties. However, long-term problems due to fatigue, excessive laxity, or wear are often reported, along with stress shielding of the new tissue and lack of host tissue integration. As a result, these devices have resulted in only minor or no improvement in clinical outcomes. ${ }^{[2,4,7,12-16]}$ The described shortcomings could potentially be solved with the use of biodegradable materials. ${ }^{[13]}$ The most popular biodegradable polymers used in clinical, including in implantable applications have been synthetic aliphatic polyesters, such as polylactic acid (PLA), poly(glycolic acid) (PGA), or poly(e-caprolactone) (PCL), for example. However, their poor mechanical properties restrict their successful use in the long term in such demanding load-bearing applications. ${ }^{[16,17]}$ 
Due to the present demand, research and engineering interests have been shifting from traditional polymeric materials to polymer-based composites. These consist of a polymeric matrix and reinforcement materials that, when combined, enable the production of advanced materials with multifunctional requirements, enhanced properties, and remarkable performances. ${ }^{[18,19]}$ In this way, the integration of reinforcing materials may overcome the limitations of a basic biodegradable polymeric device, as it can form a composite with the combinatorial benefits of each biomaterial, including biocompatibility, biodegradability, and high mechanical properties. ${ }^{[20]}$

The use of different fillers for the enhancement of the polymer's properties has been well documented. ${ }^{[21-27]}$ Yet, graphene has been one of the most studied, especially regarding the structural reinforcement of polymers, with dramatic changes in mechanical properties being reported at very small loadings. ${ }^{[28-31]}$ Furthermore, some authors report enhanced biocompatibility and biofunctionality in both in vitro and in vivo animal studies when these materials are incorporated in scaffolds for other tissue engineering applications, which makes this a very interesting approach for the envisaged purpose. ${ }^{[32,33]}$

With the emergence of this new generation of highperformance graphene-based materials, there could be now real potential to overcome the limitations of current devices and improve clinical outcomes of tendon and ligament repair. To our knowledge, no document reviewing the potential of these composite materials in this area exists. This will be our primary focus. To set the stage, the tissues' basic structure and properties are presented, followed by the description of the most used polymeric matrices for biomedical applications and the prominent challenges of current strategies, providing foundation for the need of novel approaches. Then, a brief section on graphene as a reinforcing material is presented, and its effect on the mechanical properties, biodegradability, and biocompatibility of polymer composites is addressed, always focusing on the establishment of a comparison with the envisaged application. The article will close with some possibilities and challenges that remain to be faced by future works.

\section{Tendon and Ligament Tissues}

To design scaffolds capable of properly repairing tendon and ligament injuries, it is important to understand these tissues' morphology and mechanical behavior, as well as their mechanism of injury and healing process. ${ }^{[34]}$

\subsection{Structure and Function of Tendons and Ligaments}

Tendons and ligaments are fibrous dense connective tissues. Tendons bind muscle to bone while ligaments bind bone to bone, and together, these tissues contribute to the transmission of forces that allow body movement and stabilize the skeleton. ${ }^{[3,35-38]}$ Although both tissues vary in size, shape, organization, and anatomical location, their basic structure is quite similar. They are characterized by the presence of an abundant extracellular matrix (ECM), which is mainly composed of aligned crimped collagen fibers embedded in a viscous ground substance, but a few embedded fibroblastic cells are also present, being responsible for the production of the ECM components. The most abundant fibrous component of the ECM is type I collagen, which confers strength and stiffness to the tissue to resist tension. A small fraction of elastin is also present to ensure tissue extensibility and recover after stretching. The ground substance, which consists of water, glycosaminoglycans, proteoglycans, and glycoproteins, provides structural support and is essentially responsible for the viscoelastic properties of the tissue. ${ }^{[1,3,39,40]}$

These tissues are organized in hierarchical structures of increasing complexity (Figure 1). Tropocollagen is the basic structural unit of collagen molecules. These molecules are assembled into fibrils, which consecutively assemble into fibers. These collagen fibers are organized in a regular parallel manner and are associated in fascicles (or fiber bundles), being involved by a loose connective tissue sheath. The final tissue unit, composed of grouped fascicles, is surrounded by another thin layer of connective tissue. These sheaths allow the fiber groups to glide on each other in an almost frictionless manner and typically house the vasculature and innervation of the tendon/ligament unit. Ultimately, some tissues are surrounded by another loose connective tissue layer, which facilitates gliding on adjacent structures. ${ }^{[1,2,14,40,41]}$

Due to their composition and highly aligned organized structure, these tissues present a nonlinear and anisotropic response to load, exhibiting high mechanical strength to withstand large tensile forces, good flexibility, and viscoelasticity, meaning a time-dependent and load history dependent behavior, exhibiting stress relaxation, creep, and hysteresis, with the possibility to resist the repeated cyclic strains typically experienced during daily activities, without damage. ${ }^{[2,40,42-44]}$ A typical stress-strain curve of these tissues presents an initial "toe region" $(\approx 2 \%$ strain), in which the tissue accommodates to the applied load by straightening its crimp pattern. This crimp acts as a buffering system to shock, enabling the tissue to distend without sustaining damage when a small elongation occurs. In case of further elongation, the load is directly taken up by the completely straightened collagen fibers, so the tissue enters in a linear relationship between load and strain, and the stiffness remains constant as a function of elongation. Tendons and ligaments can be strained to about $4 \%$, returning to its original length and configuration without structural damage when unloaded, undergoing elastic deformation. However, this resistive capability is not infinite, and if this physiological limit is exceeded, the tissue loses its crimp formation, becoming plastically deformed, and is vulnerable to microscopic and macroscopic tearing. ${ }^{[2,3,37,40,43-45]}$

Although the mechanical properties of a tendon/ligament tissue depend on its thickness and collagen content which, in turn, vary with its function and with patient age and condition, in general, these tissues present average values of the tensile strength

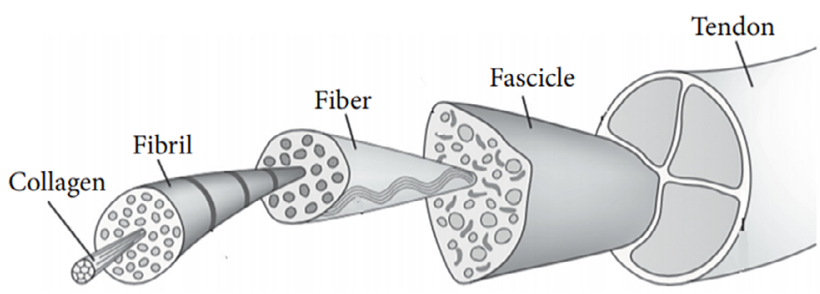

Figure 1. Structural hierarchy of a tendon. ${ }^{[16]}$ 
of 50-150 MPa, Young's Modulus in the range of 1-2 GPa, an average stiffness of $200 \mathrm{~N} \mathrm{~mm}^{-1}$, and ultimate strain values of $4-10 \% .{ }^{[2,37,40,43]}$

\subsection{Tendons and Ligaments Injury and Healing}

Several pathologies can affect tendon and ligament tissues, including degenerative disorders persistent over time, that arise from collagen damage and cellular, vascular, and neurological alterations, chronic overuse injuries resultant from previous damage accumulation as a consequence of an imbalance between the rate of microdamage occurrence and repair, and traumatic acute injuries resultant of excessive mechanical loading. ${ }^{[3,46]}$ Nevertheless, statistical data suggest that the most frequently damaged tissues are usually the ones that are exposed to the highest stresses and repeated strains and the ones that are less vascularized. ${ }^{[1,2]}$ Achilles tendon is the most frequently ruptured tendon in humans, with most ruptures occurring during sports. ${ }^{[2,47,48]}$ In Europe, an incidence rate of 37.3 cases per 100000 individuals is reported. ${ }^{[2]}$ Injuries to the glenohumeral ligaments and the rotator cuff tendons of the shoulder joint have also a high incidence, affecting more than $50 \%$ of individuals older than 60 years old. ${ }^{[36]}$ The reconstruction of the anterior cruciate ligament (ACL) of the knee is one of the most commonly performed orthopedic procedures, with around 250000 surgeries being performed annually in the US. ${ }^{[4]}$

These tissues do not heal by tissue regeneration, but through a very slow and often inefficient wound repair process instead, in which the defect is filled with scar-like tissue. ${ }^{[2,9,50]}$ This healing process involves three sequential phases: inflammation, proliferation, and remodeling/maturation (Table 1). These phases can overlap and the duration and efficiency of each one are dependent on the location and severity of the injury, namely the tissue loss degree, and on the age and condition of the patient. ${ }^{[1,2,50]}$ In the end, the composition, structural organization, and mechanical properties of the tissue are not restored completely. ${ }^{[2,3,6,9,51]}$ The healed tissue presents poor viscoelasticity and is usually weaker. ${ }^{[7,38]}$ Indeed, as shown in Figure 2, some studies indicate that tensile strength is restored only up to $80 \%$ of that of the intact tissue after a year of remodeling. ${ }^{[37,43,44]}$ As a result, it

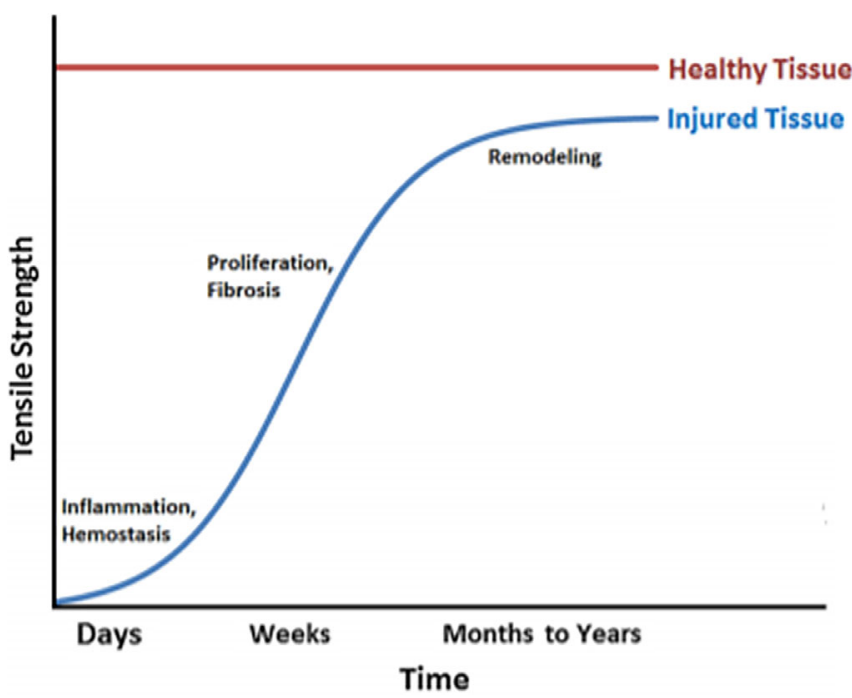

Figure 2. Evolution of the mechanical properties of a tendon during healing. Reproduced with permission. ${ }^{[2]}$ Copright 2015, Springer Nature.

presents inferior quality and functional activity, with high chances of a reoccurring rupture..$^{[1,3,7,40]}$

\section{Available Products for Tendon and Ligament Repair and Replacement}

Due to the limited ability for self-healing of tendons and ligaments, and the urgency of injured people to recover their functionality, surgical intervention is often conducted. ${ }^{[2,7]}$ Primary repairs involve the use of sutures to reattach the injured ends. However, the remaining tissue is often plastically deformed, being incapable of sustaining the rigors of normal activities and, as such, it may fail again. Furthermore, this approach is not viable in the case of extensive tissue loss. ${ }^{[3,53]}$ In such cases, the use of autografts or allografts is often required for reconstruction surgery. Both alternatives present good initial mechanical strength and allow integration within the host environment,

Table 1. Healing process of tendons and ligaments. ${ }^{[1,2,41,43,50-52]}$

\begin{tabular}{|c|c|c|}
\hline Phase & Duration & Events \\
\hline \multirow[t]{3}{*}{ Inflammation } & Hours to a few days & Hematoma formation. \\
\hline & & Migration of inflammatory cells to the injury site and cleaning of necrotic materials by phagocytosis. \\
\hline & & $\begin{array}{l}\text { Release of vasoactive and chemotactic factors resulting in the formation of a vascular network and in the } \\
\text { recruitment of fibroblasts to initiate synthesis and deposition of ECM components, respectively. }\end{array}$ \\
\hline \multirow[t]{4}{*}{ Proliferation } & Weeks & Synthesis and deposition of collagen type III and other ECM components. \\
\hline & & Formation of thinner, poorly aligned fibrils with fewer crosslinks, giving rise to a disorganized fibrous tissue \\
\hline & & The new tissue is highly cellular and contains large amounts of water. \\
\hline & & The healing tissue remains mechanically weak and vulnerable to reinjury. \\
\hline \multirow[t]{4}{*}{ Remodeling/maturation } & Months to years & Decrease in cellularity and matrix production. \\
\hline & & The proportion of collagen type I increases. \\
\hline & & $\begin{array}{l}\text { Covalent bonding between collagen fibers increases. Both fibers and tenocytes align with the direction of stress, } \\
\text { resulting in the increase of tissue stiffness. }\end{array}$ \\
\hline & & Fibrous tissue slowly transforms into scar-like tissues. \\
\hline
\end{tabular}


but long-term rupture and excessive laxity are reported causes of mechanical failure. Furthermore, material selection and dimensioning can be problematic. ${ }^{[13]}$ While autografts present disadvantages as limited availability and require additional surgery which causes increased donor site morbidity and recovery time, allografts present associated risks of immune rejection or disease transmission. ${ }^{[2,5,8,9,14,37,54]}$

In an attempt to improve clinical outcomes where these standard repair strategies failed, several biological and synthetic scaffold devices were developed (Table 2). These scaffolds were produced to aid the restoration of normal function temporarily or permanently. Most of these devices, mainly in the form of patches, have been used for mechanical augmentation. They are either used to provide immediate protection after a small tissue tear by sharing mechanical stresses with the injured tissue, allowing the original repair to proceed without suffering excessive disruptive loads, or can be also combined with grafts to provide additional structural and mechanical support. Only a few synthetic nondegradable devices have been used to fully replace the tissue in case of extensive tissue loss, although no reliable artificial tendon/ligament exists. ${ }^{[2,4,9,14,55,56]}$

\subsection{Biological Scaffolds}

Biological scaffolds consist of human or animal (equine, porcine, and bovine) tissues, as pericardium, dermis, or small intestine submucosa (SIS), which are processed to remove noncollagen components that may cause host rejection, while preserving their natural collagen structure. Because of their natural composition and inherent porosity, these scaffolds can provide a chemically and structurally instructive environment, providing a better and faster host cell integration, thus having a marked effect on the biology of healing events. However, the decellularization method may present drawbacks, such as the presence of trace chemicals which can be toxic to host cells. In addition, these scaffolds may present variation in biocompatibility, undefined degradation rates, and low mechanical properties, not being adequate to bear substantial loads. Indeed, these scaffolds are often provided in numerous layers to increase the mechanical properties of the product in an attempt to realistically provide mechanical reinforcement to the repair site. ${ }^{[2,4,11,55-58]}$

\subsection{Synthetic Scaffolds}

Due to the low mechanical properties of biological scaffolds, distinct synthetic scaffolds, manufactured from synthetic biodegradable and nonbiodegradable polymers, have been developed. Besides the improved mechanical properties, synthetic polymers also offer other advantages linked to large-scale manufacturing, as they present a reliable source of raw materials along with low immunogenic risks, are available in a wide variety of compositions, and have an attractive processability, as they can be

Table 2. Commercially available products for tendon/ligament tissue repair. ${ }^{[2,4,11,14,55,56]}$

\begin{tabular}{|c|c|c|c|c|}
\hline Category & Product name & ECM type & ECM source & Marketed by \\
\hline \multicolumn{5}{|l|}{ (a) Extracellular-Matrix } \\
\hline \multirow[t]{9}{*}{ Augmentation device } & Restore & SIS & Porcine & Depuy Orthopaedics (USA) \\
\hline & CuffPatch & SIS (cross-linked) & Porcine & Arthrotek (USA) \\
\hline & GraftJacket & Dermis & Human & Wright Medical (USA) \\
\hline & ArthroFlex & Dermis & Human & Arthrex (USA) \\
\hline & Conexa & Dermis & Porcine & Tornier (USA) \\
\hline & TissueMend & Dermis (fetal) & Bovine & Stryker Orthopaedics (USA) \\
\hline & Zimmer Collagen Repair & Dermis (cross-linked) & Porcine & Zimmer (USA) \\
\hline & Bio-Blanket & Dermis (cross-linked) & Bovine & Kensey Nash (USA) \\
\hline & OrthADAPT Bioimplant & Pericardium (cross-linked) & Equine & Pegasus Biologics (USA) \\
\hline Category & Product name & \multicolumn{2}{|c|}{ Material } & Marketed by \\
\hline \multicolumn{5}{|l|}{ (b) Synthetic } \\
\hline \multirow[t]{8}{*}{ Augmentation device } & SportMesh & \multicolumn{2}{|c|}{ Poly(urethane urea) } & Biomimet Sports Medicine (USA) \\
\hline & X-Repair & \multicolumn{2}{|c|}{ Poly-L-lactide } & Synthasome (USA) \\
\hline & Biomerix Patch & \multicolumn{2}{|c|}{ Polycarbonate poly(urethaneurea) } & Biomerix (USA) \\
\hline & Gore-Tex & \multicolumn{2}{|c|}{ Poly(tetrafluoroethylene) } & WL Gore and Associates (USA) \\
\hline & Kennedy Ligament & \multicolumn{2}{|c|}{ Polypropylene } & DAW Industries, 3M (USA) \\
\hline & Leeds-Kuff Patch & \multicolumn{2}{|c|}{ Polyester } & Neoligaments (UK) \\
\hline & Rota-Lok & \multicolumn{2}{|c|}{ Polyester } & Neoligaments (UK) \\
\hline & AchilloCordPLUS & \multicolumn{2}{|c|}{ Poly(ethylene terephthalate) } & Neoligaments (UK) \\
\hline \multirow[t]{3}{*}{ Prosthetic device } & OrthoCoupler & \multicolumn{2}{|c|}{ Poly(ethylene terephthalate) } & Surgical Energetics (USA) \\
\hline & Lars Ligament & \multicolumn{2}{|c|}{ Poly(ethylene terephthalate) } & Lars (France) \\
\hline & Leeds-Keio & \multicolumn{2}{|c|}{ Poly(ethylene terephthalate) } & Neoligaments (UK) \\
\hline
\end{tabular}


fabricated into different forms with lot-to-lot uniformity and predictable, controllable, and reproducible chemical, mechanical, and degradation properties. ${ }^{[2,59-64]}$ Furthermore, they can be modified to solve problems as inflammatory side effects, hydrophobicity, and low cell adhesion. ${ }^{[65]}$

The first attempts that have been made for tendon and ligament repair used nondegradable synthetic polymers such as poly(ethylene terephthalate) (PET), used in devices as the Lars Ligament (Lars, France) or the Leeds-Keio (Neoligaments, UK), or polypropylene (PP) as in the Kennedy Ligament device (DAW Industries, USA), due to the desired properties of excellent tensile strength and biocompatibility. Most of these devices have been used for mechanical augmentation and only a few were intended for a permanent and complete replacement of the tissue. Although these devices can initially replace the function or protect the tissue that they are meant to repair, their inability to mimic the native tissues' mechanical behavior has led to failure under loading and poor long-term results. As a result, some are no longer used or even approved in some countries. ${ }^{[2,7,12-14,54,56]}$ Tendon and ligament tissues are continuously subjected to mechanical loads during body movements, a demanding mechanical environment that restricts the successful application of most devices alone. Common observed problems include device rupture due to fatigue, excessive laxity from repeated elongation and creep, and lack of durability related to wear and material degeneration, along with inflammatory responses associated with the produced wear debris. Stress shielding of the new formed tissue and limited integration between the device and host tissue resulting in foreign body reactions are also reported problems associated with the use of these permanent devices. ${ }^{[2,14,54,56,66]}$

In view of these problems, it was logic to consider the use of biodegradable polymers for the development of these devices. ${ }^{[17]}$ A few synthetic biodegradable augmentation devices exist, based on poly(urethane urea) as the SportMesh patch (Biomimet Sports Medicine, USA) and on poly(lactic acid) (PLA) as the X-Repair patch (Synthasome, USA), for example, but are not intended to replace the tissue structure or provide full mechanical strength to support the repair. ${ }^{[67]}$

\section{Designing Novel Tendon and Ligament Tissue Substitutes: Application of Biodegradable Matrices}

Owing to the limitations associated with current approaches, there has been an increasing market interest to develop a suitable system that is able to promote/accelerate healing and re-establish the native structure and functionality of an injured tendon/ ligament. ${ }^{[7,68-70]}$

An ideal scaffold meant for tendon and ligament repair should be a biocompatible and biofunctional porous structure able to promote and support tissue formation, without causing inflammation or other reactions that may reduce or impede the healing process or cause rejection by the body. It should present adequate initial strength and mimic the nonlinear and viscoelastic response of the native tissues, to adequately support the repair and substitute the mechanical function of the diseased or damaged tissue during the necessary healing period. This should be accomplished while presenting a gradual degradation, during a period that approximately matches the regrowth rate of the new forming tissue, to concede room for new tissue growth and integrate within the host environment and allow a controlled exposure of the new tissue to the local mechanical environment, avoiding a stress-shielding phenomenon..$^{[2,35,63,64,71]}$ It is well demonstrated that controlled mechanical loads enhance the quality of the healing process, namely of repair and remodeling events, by stimulating fibroblastic proliferation, differentiation, collagen synthesis, and realignment, which is essential for a homogeneous and functional repair. ${ }^{[39,45,72]}$

The selection of biomaterial is, therefore, a key element for the development of tissue-repair devices. ${ }^{[64]}$ Current biodegradable biomaterials used in scaffold fabrication can be divided into categories of synthetic or naturally derived. ${ }^{[73]}$

Natural scaffolds include collagen, silk, hyaluronic acid, chitosan, or fibrin, for example. Despite their higher biofunctionality and biocompatibility, having the ability to be biologically recognized and provide better environment to support the biological events of tissue healing, these materials present limitations that limit their application and evolution from a research stage to the clinic, at least for now. These include their high induction of antigenic and immunogenic reactions, low mechanical properties, not predictable or controlled degradation kinetics as their degradation depends on availability and concentration of enzymes in the implantation site, difficult processing, high cost, and variety from batch to batch. ${ }^{[2,9,16,71,74]}$

On the other hand, synthetic polymers have been widely applied in the medical field, which is essentially due to the aforementioned advantages related to their properties and processability. Polyesters such as poly(glycolic acid) (PGA), the stereoisomer forms of poly(lactic acid), L-PLA and D-PLA, and their copolymer poly(lactic-co-glycolide) (PLGA) and poly(e-caprolactone) (PCL), are among the most attractive biodegradable polymers used in biomedical applications. These materials are already approved by the Food and Drug Administration (FDA) for clinical use, including in implantable applications, are considered biocompatible and degrade by hydrolysis into nontoxic components that are usually metabolized or excreted by the body. ${ }^{[62,64,65,75-77]}$ Due to these reasons, dozens of synthetic biodegradable medical devices are commercially available, and every year more have been introduced to the market. Some examples include fracture fixation screws, sutures, dental orthopedic implants, stents, vascular grafts, wound healing and surgical meshes, drug delivery, and tissue augmentation. ${ }^{[65,75,78]}$ The use of biodegradable polymers for other applications is also being pursued relentlessly on a research level. ${ }^{[17]}$ These include scaffolds for tendon/ligament direct replacement or tissue-engineering applications (Table 3).

Nevertheless, biodegradable synthetic polymers still present a number of disadvantages that have to be addressed, as they have limited the further application and evaluation of these materials in animal and human trials. ${ }^{[17,88]}$ For instance, these materials are mechanically weaker than healthy musculoskeletal tissues and, as such, do not reach the required in vivo strength. ${ }^{[16,17]}$ It is unquestionable that mechanical conditions are a key role in material performance, especially in load-bearing applications, and that the lack of mechanical compatibility between the device and the native tissue interferes with the natural healing mechanism and can thus represent a serious drawback for the damaged tissue repair. ${ }^{[65]}$ Also, it is difficult to achieve a balance between 
Table 3. Examples of synthetic biodegradable scaffolds that have been studied for potential tendon/ligament repair strategies.

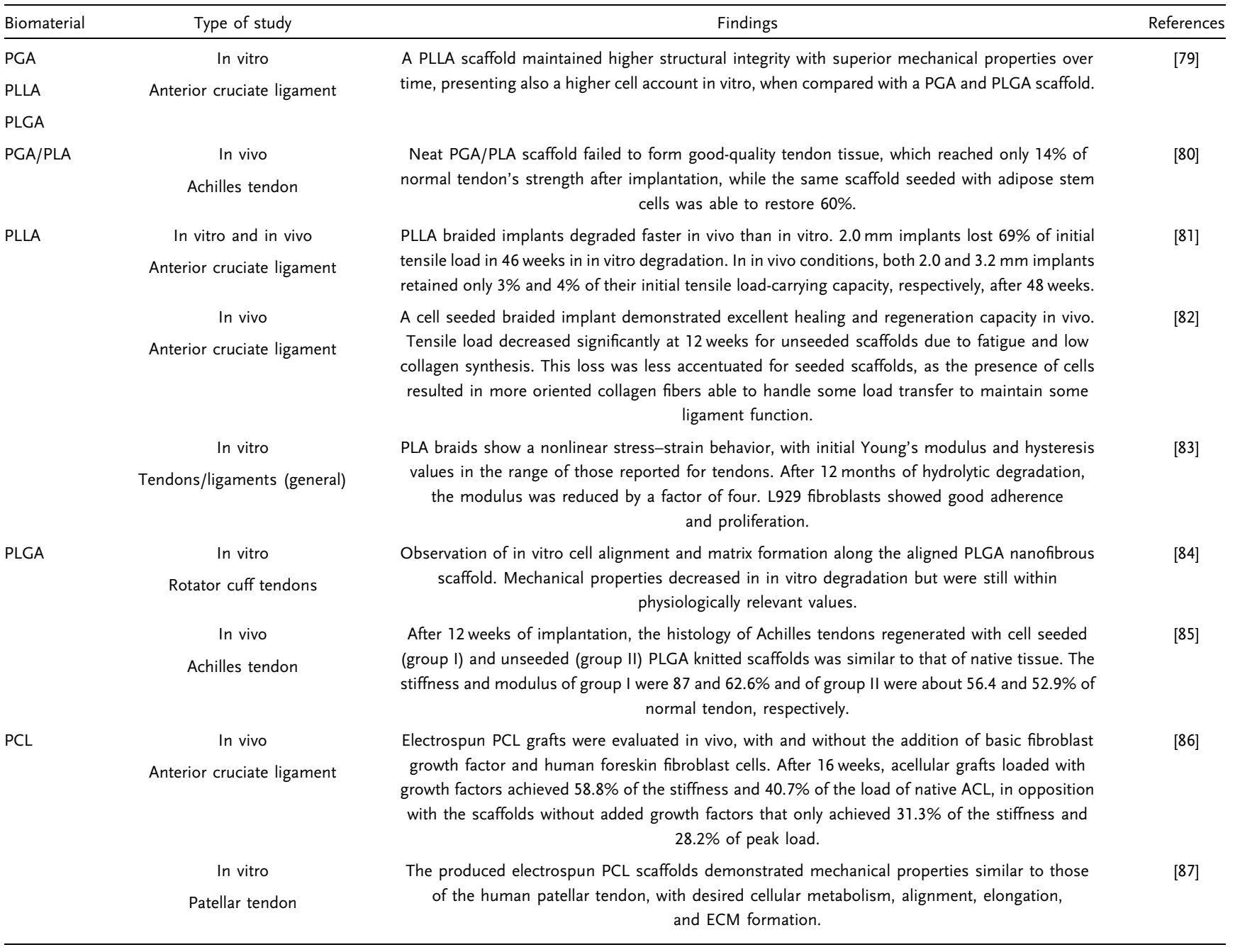

Table 4. Properties of some synthetic polymers approved for use in implantable medical devices in comparison with tendon/ligament tissues. $\mathrm{T} / \mathrm{L}$-tendon/ligament tissues (general values); $\varepsilon$ - elongation at break. ${ }^{[43,65,75,89-91]}$

\begin{tabular}{lccccc}
\hline Polymer & $\begin{array}{c}\text { Tensile } \\
\text { strength } \\
{[\mathrm{MPa}]}\end{array}$ & $\begin{array}{c}\text { Young's } \\
\text { modulus } \\
{[\mathrm{GPa}]}\end{array}$ & $\varepsilon[\%]$ & $\begin{array}{c}\text { Loss of } \\
\text { strength } \\
\text { [months] }\end{array}$ & $\begin{array}{c}\text { Loss of } \\
\text { mass } \\
\text { [months] }\end{array}$ \\
\hline PGA & $55-80$ & $5-7$ & $15-20$ & $1-2$ & $6-12$ \\
PLLA & $45-70$ & $2.7-3.5$ & $5-10$ & $3-6$ & $>24$ \\
PLCA & $55-80$ & 2 & $3-10$ & 1 & $1-12$ \\
PCL & $10-35$ & $0.4-0.6$ & $300-500$ & $>6$ & $>24$ \\
T/L & $50-150$ & $1-2$ & $4-10$ & - & - \\
\hline
\end{tabular}

material degradability/loss of strength and the rate of tissue healing and matrix production (Table 4). A gradual degradable device, that still preserves at least half of its initial strength for at least 3-6 months implantation, is highly recommended for adequate mechanical support in tendon/ligament repair. ${ }^{[92]}$ This class of materials undergoes a notable decrease in mechanical strength after implantation ${ }^{[93]}$ and are not able to maintain tensile integrity during the necessary healing period, which resulted in the device's early failure. ${ }^{[68,88,94]}$ Besides their low tensile strength, they are also susceptible to creep and fatigue failure under dynamic loading. ${ }^{[93,95]}$ Moreover, polymers such as PLA or PGA still present another challenge related to their acidic degradation byproducts, which are known to reduce the local $\mathrm{pH}$ of surrounding tissues, accelerate degradation, and induce inflammatory reactions. ${ }^{[13,65,93]}$ The formation of blends, using different polymers at adjustable concentrations, has been used in an attempt to adjust mechanical properties and/or degradation rate but still demonstrates limitations. ${ }^{[16,70]}$

Even though over the years more information has become available, the effectiveness of a particular polymer for tendon and ligament repair is still a matter of discussion. The scarce number of studies that exist reviewing biodegradable devices report mixed results and lack definitive conclusions, but it is acknowledged that conventional single-component biodegradable polymer materials 
cannot satisfy all the requirements for tendon/ligament repair. As a result, no biodegradable replacement has been approved for clinical use. ${ }^{[14,25,70,74,96,97]}$ Thus, these biodegradable devices have to be optimized to provide appropriate mechanical support during the necessary period. ${ }^{[54]}$

As research for alternative ways continues, it is expected that, in the future, novel strategies will lead to the design and production of next-generation structures, mimic the mechanics of native tendon/ligament tissues, and have the potential to lead to the complete formation of a new, mechanically adequate natural tissue. ${ }^{[54]}$ Among these, the design and preparation of innovative polymer composites is considered one of the most stimulating strategies. The introduction of reinforcement materials into biocompatible and biodegradable polymers could be an important possibility to increase and modulate mechanical and degradation properties, thus obtaining a material that fulfills several advanced functions at once, overcoming the limitations of current devices. ${ }^{[25,60,70,98]}$

\section{The Potential of Novel Graphene-Polymer Composites for Tendon and Ligament Repair}

The field of polymer composites has been growing, opening up new possibilities in the development of advanced materials for various modern applications, including in the biomedical and biotechnological field, where conventional polymers cannot meet all performance requirements. ${ }^{[2,99-102]}$ These novel structures can be defined as a multicomponent combination of two or more materials, a discontinuous or dispersed phase called reinforcement, and the polymeric matrix, a continuous phase surrounding the reinforcement, which, when combined, produce a final composite that exhibits a combination of properties of their original components, but may also acquire new properties, which are not depicted by any of the constituents alone. ${ }^{[102-104]}$

A wide variety of reinforcements can be incorporated within a polymer network to provide enhanced functionality, and the choice depends on several factors such as cost, processability, and the target properties. ${ }^{[21-26,102]}$ Metal particles such as gold, silver, iron, or copper, for example, may improve electrical, optical, magnetic, and electrochemical properties while metal-oxide particles such as titanium dioxide or zinc oxide demonstrate good properties to act as antibacterial agents. ${ }^{[22-26]}$ Calcium phosphates and bioglass ceramic particles have been the most extensively studied and used materials for bone tissue engineering due to their osteoconductive nature. ${ }^{[22,25,100,101]}$ On the other hand, carbon-based materials such as carbon nanotubes (CNTs) and graphene have been used in the production of polymer composites as mechanical reinforcement agents. ${ }^{[21-26]}$ However, in comparison with CNTs, graphene presents advantages such as lower production and purification costs and higher surface-to-volume ratio. ${ }^{[28,30,105-107]}$ Moreover, it has been shown that, within the same loading, graphene-reinforced composites may even outperform single-/multiwalled CNT-reinforced composites in mechanical properties. ${ }^{[30,108,109]}$ Furthermore, the tubular morphology of CNTs, with nanometric diameter and a high aspect ratio, appears as a potential risk to human health, thus limiting their application in biomedical areas. Conversely, graphene with its 2D morphology and nanometric thickness has demonstrated good biocompatibility. ${ }^{[110]}$
This distinctive combination of properties makes graphene one of the most promising materials to improve the performance of conventional polymers, giving them the possibility to be used in several fields for novel applications where their use is normally restricted, including in tendon/ligament repair. As such, this material will be the topic of further discussion in the next sections.

\subsection{Graphene as Reinforcement: Properties and Composite Processing}

Nowadays, graphene is considered the most attractive material in the world of nanotechnology. ${ }^{[111]}$ It is a 2D planar single layer of $\mathrm{sp}^{2}$ hybridized carbon atoms, densely arranged in a hexagonal lattice (Figure 3). These carbon atoms are bonded together by strong covalent $\sigma$-bonds ( $\mathrm{C}-\mathrm{C}$ bonds), with an additional outof-plane $\pi$-bond, forming a conjugated $\mathrm{C}=\mathrm{C}$ network and leading to a bond length of $0.142 \mathrm{~nm}$. Graphene has a thickness of $0.34 \mathrm{~nm}$, which makes it the material with the smallest thickness known. ${ }^{[105,112-116]}$

This unique planar arrangement and geometry of graphene, with the strong carbon-carbon bonding, the aromatic structure, and the presence of free $\pi$ electrons, makes it a stable material with unique characteristics, such as light weight, a high surface area $\left(2630 \mathrm{~m}^{2} \mathrm{~g}^{-1}\right)$, high elastic modulus $(\approx 1 \mathrm{TPa})$, and high tensile strength $(130 \pm 10 \mathrm{GPa})$. Furthermore, graphene also presents excellent thermal conductivity $\left(5000 \mathrm{~W} \mathrm{~m}^{-1} \mathrm{~K}^{-1}\right)$, high electron mobility at room temperature $\left(250000 \mathrm{~cm}^{2} \mathrm{~V}^{-1} \mathrm{~s}^{-1}\right)$, an optical transmittance of $98 \%$, and a tunable surface chemistry that opens up many possibilities to obtain useful chemical functionalization. ${ }^{[107,114,116-119]}$

A number of methods for graphene synthesis based on bottom-up or top-down approaches have been emerging and allow for numerous choices in terms of size, quality, and cost. $^{[31,111,115,116,119-121]}$ Among them, the liquid-phase exfoliation (LPE) of graphite has shown to be promising for the mass production of graphene. Graphite's low cost and availability, along with the simplicity, speed, and high throughput of highquality graphene with good yield that is associated with this process, make this approach very attractive among researchers and

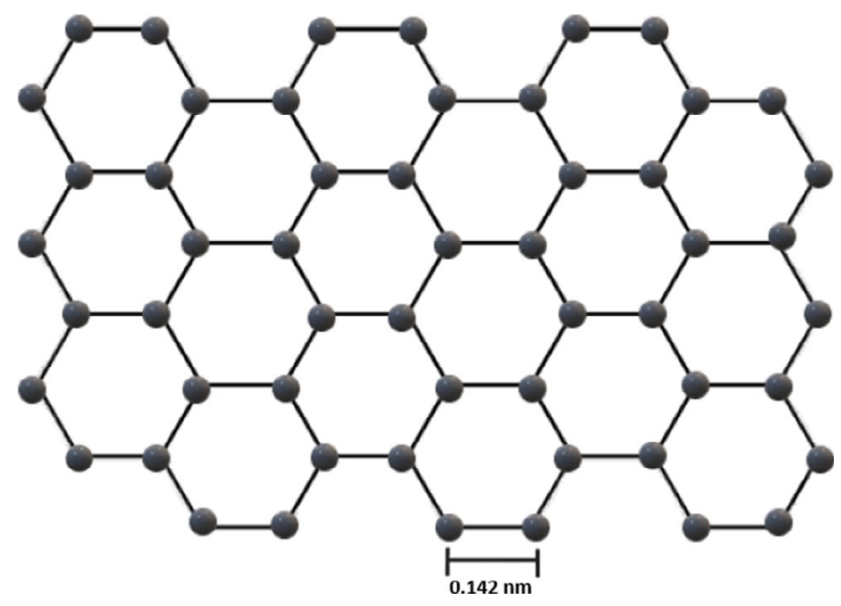

Figure 3. Structural representation of graphene. 
promising for large-scale production. ${ }^{[31,116,122,123]}$ However, the production of defect-free monolayer graphene is still laborious and costly for industrial applications. As a result, related materials such as few-layer graphene (FLG) (2-10 layers of graphene), ultrathin graphite ( $\geq 10$ layers but less than $100 \mathrm{~nm}$ thick), and graphene nanoplatelets (GNPs) (composed of single-layer and few-layer graphene mixed with thicker graphite), which are commonly obtained following the LPE procedure without further isolation steps, have been used instead. ${ }^{[109,121,124]}$

Graphene's unique combination of properties indicate its great potential for improving the thermal, electrical, and mechanical properties of polymer composites. In particular, this material has attracted enormous interest for the mechanical reinforcement of polymers, as extraordinary improvements have been reported in the presence of a variety of polymeric matrices, even with a small amount of graphene. ${ }^{[2-31,125]}$ As a result, the present composite materials have led to a new generation of advanced materials, opening up a wide range of novel applications in several fields, such as in electronics, aerospace, automotive, construction, and biomedical. ${ }^{[28,109,126-129]}$

A crucial step in polymer composites is the dispersion of the reinforcement in the polymer. ${ }^{[109]}$ A filler with a large surface area-to-volume ratio in a well-dispersed state ensures a maximized interfacial area, which will enhance the load-transfer ability of the mechanical force from the polymer to the reinforcing phase and consequently, improve the properties of the composite. ${ }^{[21,130-132]}$ However, graphene, like all nanomaterials, has a pronounced tendency to agglomerate in a polymer matrix. ${ }^{[30,118,125]}$ These nanoparticle agglomerates are unable to interact efficiently with the polymeric matrix, prevent a uniform load transfer, and can even act as stress concentration points, resulting in inferior properties or only mild performance improvement, thus sacrificing graphene's efficiency as reinforcement. ${ }^{[21,118,125,132]}$

Large efforts have been concentrated on achieving a homogeneous and well-dispersed system via a compatibilization process, which includes covalent or noncovalent functionalization of the filler surface. ${ }^{[30,118,133]}$ Covalent functionalization involves partial modification of the graphene $\mathrm{sp}^{2}$ carbon lattice, leading to covalent bonding of chemical functional groups to the graphene surface. It may result in heteroatomic irregularity as well as introduction of impurities and structural defects. This destruction of graphene's original structure can lead to impaired properties. ${ }^{[30,105,107,117,120,128]}$ The most common example involves the oxidation of graphene into graphene oxide (GO) to increase hydrophilicity and facilitate dispersion and preparation of composites. However, the presence of oxygen groups generates structural defects that, in turn, affect strongly the mechanical and electrical properties compared with graphene. ${ }^{[31,106,107,117]}$ Noncovalent modifications involve secondary interactions or physical adsorption/wrapping of specific compounds on the graphene surface. This approach generally relies on weak interactions and preserves graphene's structural integrity, imparting no structural damage, as it does not affect the $\mathrm{sp}^{2}$ carbon hexagonal lattice of graphene and does not disturb the intrinsic properties of the material, being more advantageous from that point of view. ${ }^{[30,105,107,117,120,128]}$

The ultimate properties of graphene-polymer composites also depend on the composite processing conditions. The most popular processes have been either solution mixing or melt mixing. Solution mixing involves the dissolution of the polymer and suspension of graphene in the same or miscible solvents, followed by their mixing with the use of stirring, ultrasonication, or shear mixing to disperse the particles homogeneously, before removing the solvent. This approach is simple, with fast fabrication and high control on component behavior, and very effective for dispersing the filler in the matrix. However, challenges include finding common solvents, avoiding toxic and non-ecofriendly organic solvents, which can be costly in largescale applications, difficulties in solvent removal, and common reagglomeration during solvent evaporation. These observations make apparent the challenges and limitations of graphene chemistry in solvent-mixing procedures and justify why this method gets less attention for industrial applications. Melt mixing is a solvent-free process that involves melting the polymer at an adequate temperature and mixing graphene (in a powder form) at high mechanical shear conditions. Compared with the previous method, this approach is simpler, faster, economically advantageous, environmentally friendly, and more compatible with many current industrial productions. However, the viscosity of the polymer may result in less-effective dispersion of the filler at higher loadings. ${ }^{[21,31,107,121,128,134]}$

\subsection{Graphene-Polymer Composites for Tendon/Ligament Repair}

Graphene and graphene-based materials have attracted interest in several biomedical applications, including biosensing and bioimaging, drug delivery systems and gene therapy, photothermal therapy, and as antibacterial agents. ${ }^{[114,121,135-138]}$ Methodologies for their incorporation in polymers for different tissueengineering strategies (e.g., cardiac, neural, cartilage, bone, musculoskeletal tissue, skin) have also been under study, majorly focusing on its effect as structural reinforcement in hydrogels, films, fibers, and other tissue-engineering scaffolds, to optimize the primary mechanical properties of the neat materials that are currently being used for this purpose, which are often below the requirements. ${ }^{[32,33,110,121,136]}$ These scaffolds can even exhibit other functional properties of interest, such as enhanced electrical conductivity. It is acknowledged that electrical conductivity can be a useful tool to direct cell growth, enhance cellular activities, and stimulate the tissue-healing process. ${ }^{[106,110,136]}$ Furthermore, graphene and graphene-related materials are considered exceptional substrates for promoting the adhesion, proliferation, and differentiation of various cell types and thus, facilitating the tissue-reforming process. ${ }^{[33]}$

The addition of graphene and graphene-based materials into biodegradable matrices may be an interesting strategy to improve the performance of a tendon/ligament biodegradable scaffold. The goal is to obtain properties comparable with the traditionally used nondegradable polymers and the native tissues, to match the required properties to provide the necessary support during the repair process. However, to effectively understand the impact that graphene may have in the modulation of tendon/ligament scaffold properties and its potential for the repair of these tissues, its effect on several parameters should be carefully evaluated. Namely, the mechanical properties, biodegradability, and biocompatibility of 
the matrix are of great importance in determining the success of the final outcome and may be sensitive to filler incorporation. Therefore, the effect of these materials upon the behavior of the most interesting and researched biodegradable polymers for tendon and ligament repair will be reviewed next.

\subsubsection{Effect on Mechanical Properties}

Tensile Mechanical Properties: Most of the work on graphene/ polymer composites is aimed at improving the mechanical properties of the composite material. The evaluation of the reinforcing capacity is usually accomplished by the analysis of the stress-strain curves obtained by tensile testing. Hence, most research studies focus on the mechanical properties of graphenefilled polymer composites, revealing changes in tensile strength, elastic modulus, and elongation at break and toughness, as a function of filler loading. ${ }^{[29,31,109]}$

However, despite all the research conducted in this area, several problems have to be resolved and the full potential of these composites is still to be reached. The mechanical properties of these composites depend on several parameters such as the physical structure of graphene, its chemical characteristics, the composite preparation method, the dispersion of the nanoparticles in the matrix, and the final nanoparticle-matrix interactions. Although it was reported that the mechanical properties of the composite may be remarkably increased even at very small filler loadings, in other cases, deterioration of the properties is observed. The polymer's brittleness can significantly increase in the presence of nanoparticle agglomerates, which are known to act as failure points. The presence of a considerable concentration of nanoparticle agglomerates justifies the observation of tensile strength saturation or even decrease while increasing nanoparticle loading. Typically, elongation at break follows a similar trend and decreases extensively at high nanoparticle loads, whereas the tensile modulus usually increases with increasing filler content, being less susceptible to the presence of agglomerates and responding to the overall composition. However, it should be noted that these properties do not necessarily decrease with the introduction of graphene. Numerous authors have reported strength and ductility improvements as a result of improved dispersion, especially at low filler contents. ${ }^{[31]}$ The introduction of these fillers may also affect the polymer's crystallinity, as graphene may act as a nucleating agent during crystallization and enhance the degree and rate of the crystallization process of the composite, which may increase the material's strength but also restricts the movement of the polymer chains, making it brittle. ${ }^{[21,139]}$ Furthermore, as previously mentioned, the presence of functional groups may enable stronger interactions between the filler and the matrix, and so, it may result in a higher reinforcing ability. As such, functionalized materials are often preferred over neat graphene-based materials for mechanical reinforcement. ${ }^{[31,107]}$

Table 5 shows results reported in the literature on the mechanical properties of the previously mentioned biodegradable synthetic polymers, reinforced with different graphene-based materials. Only a few examples will be described in detail.

Li et al. ${ }^{[141]}$ prepared PLA/graphene composite films by solvent mixing and verified a continuous increase in tensile strength with increasing graphene loading from 0 to $1.0 \mathrm{wt} \%$, reporting a maximum increase of $39.57 \%$ for $1.0 \mathrm{wt} \%$. However, both elongation at break and toughness values decreased with increasing graphene content, meaning that although the introduction of graphene resulted in increased polymer strength, it also restricted the movement of the polymer chains, increasing its brittleness. Other authors reported similar results, such as Tong et al. ${ }^{[159]}$. Scaffaro et al., ${ }^{[148]}$ and Narimissa et al., ${ }^{[149]}$ who also verified an increase in composite stiffness to the detriment of its tensile strength and elongation at break. The addition of the reinforcement material leads to weak regions in the matrixfiller system, along with embrittlement. Pinto et al. ${ }^{[142]}$ prepared PLA/GO and PLA/GNP composite films by solvent mixing, reporting a maximum in mechanical performance for only $0.4 \mathrm{wt} \%$ loading of the two graphene-based materials tested, with an increase of $15 \%$ in tensile strength and $85 \%$ in Young's modulus, decreasing at higher loadings for the final solvent-free films. In this case, elongation at break measurements did not show a well-defined dependence on loading and seemed not to be considerably affected by filler addition. Similar behavior was reported by Gonçalves et al. ${ }^{[147]}$ who prepared biocompatible PLA/GNP films by melt mixing, reporting a maximum mechanical performance at only $0.25 \mathrm{wt} \%$ loading, with a $20 \%$ increase in tensile strength, 12\% increase in Young's modulus, and $16 \%$ increase in toughness (Figure 4). However, a decrease in mechanical performance at higher loadings was observed, which was attributed to increased nanoparticle agglomeration that introduced defects in the polymer matrix. Again, no significant differences were observed between PLA and composites regarding elongation at break.

Li et al. ${ }^{[143]}$ prepared PLLA-based composites by solvent mixing using GO and functionalized GO-g-PLLA, to study the effectiveness of functionalization in the final properties of the composite. At $0.5 \mathrm{wt} \%$ loading, the authors reported both increase in strength and ductility, namely an increase of $51.4 \%$ in tensile strength and of $37.1 \%$ in elongation at break in PLA/GO composites and an increase of $105.7 \%$ in tensile strength and of $122.8 \%$ in elongation at break for functionalized GO composites, which effectively illustrated the benefits of a compatibilization process in aiding the filler dispersion and interfacial interactions with the matrix.

The same trends were observed for composites with other polymers. Yoon et al. ${ }^{[152]}$ prepared PLGA/GO composites by solution mixing, followed by electrospinning, with 1 and $2 \mathrm{wt} \%$ filler loading. It was at maximum loading that the authors observed the highest reinforcement effect, reporting an impressive increase in 36.4-74.6 MPa in tensile modulus, 2.1-3.4 MPa in ultimate tensile stress, and $9.5-30.7 \%$ in ultimate strain. Similarly, Mohammadi et al. ${ }^{[154]}$ prepared PCL composites with different GO contents of $0.5,1$, and $2 \mathrm{wt} \%$ and it was also at maximum loading that the authors observed significant enhancement of properties. The pure PCL had a tensile strength of $1.61 \mathrm{MPa}$, an elongation of $122 \%$, and a Young's modulus of 7.01 MPa and when 2\% GO was added, a considerable increase was observed in tensile strength to $3.50 \mathrm{MPa}$, elongation to $131 \%$, and Young's modulus to $15.15 \mathrm{MPa}$. Lastly, Ramazani et al. ${ }^{[156]}$ incorporated $\mathrm{GO}$ and reduced GO at $0.1,0.5$, and $1 \mathrm{wt} \%$ into PCL, and studied the effect of both particles on the mechanical properties of the composite. The authors 
Table 5. Mechanical properties of biodegradable graphene-polymer composites. $\sigma$, tensile strength; $E$, Young's modulus; $\varepsilon$, elongation at break; NR, not reported; f, functionalized; DDAB, didodecyldimethylammonium bromide; PSS, Poly(sodium 4-styrenesulfonate).

\begin{tabular}{|c|c|c|c|c|c|}
\hline Matrix & Mixing method & Graphene type & Loading [wt\%] & Mechanical properties & Ref. \\
\hline \multirow[t]{14}{*}{ PLA } & Solution mixing & Graphene nanosheets (GNS) & 0.2 & $\sigma: \uparrow 26 \% ; E: \uparrow 18 \%$ & [140] \\
\hline & & Exfoliated graphene & 1 & $\sigma: \uparrow 39.57 \% ; \varepsilon: \downarrow(\mathrm{NR})$ & [141] \\
\hline & & GNPs and GO & 0.4 & $\sigma: \uparrow 15 \% ; E: \uparrow 85 \% ; \varepsilon:=$ & [142] \\
\hline & & GO & 0.5 & $\sigma: \uparrow 51.4 \% ; \varepsilon: \uparrow 37.1 \%$ & [143] \\
\hline & & GO-g-PLLA (f) & & $\sigma: \uparrow 105.7 \%, \varepsilon: \uparrow 122.8 \%$ & \\
\hline & Melt mixing & GO & 0.2 & $\sigma: \uparrow 8.8 \% ; E:=; \varepsilon: \downarrow 54.2 \%$ & [144] \\
\hline & & DDAB-GO (f) & & $\sigma: \uparrow 6.3 \% ; E: \downarrow 23.3 \% ; \varepsilon: \uparrow 2659.3 \%$ & \\
\hline & & Exfoliated graphene & 0.2 & $\sigma: \uparrow 6.1 \% ; E: \uparrow 6.5 \% ; \varepsilon: \downarrow 9.5 \%$ & [145] \\
\hline & & Graphene & 0.08 & $\sigma: \uparrow 35 \%$ & [146] \\
\hline & & GNP & 0.25 & $\sigma: \uparrow 20 \% ; E: \uparrow 12 \% ; \varepsilon:=$ & [147] \\
\hline & & GNP & 5 & $\sigma: \downarrow 1.9 \% ; E: \uparrow 39.4 \% ; \varepsilon: \downarrow 36.9 \%$ & [148] \\
\hline & & Nanographite platelets & 3 & $\sigma: \downarrow(\mathrm{NR}) ; E: \uparrow 135 \% ; \varepsilon: \downarrow$ (NR) & [149] \\
\hline & & Small GNPs & 5 & $\sigma: \uparrow 16 \% ; E: \uparrow 10 \% ; \varepsilon: \downarrow 3 \%$ & [150] \\
\hline & & Large GNPs & & $\sigma: \uparrow 41 \% ; E: \uparrow 24 \% ; \varepsilon: \downarrow 60 \%$ & \\
\hline \multirow[t]{2}{*}{ PLGA } & Solution mixing & GO & 5 & $\sigma: \uparrow 153.5 \% ; E: \uparrow 372.9 \% ; \varepsilon: \downarrow 53.4 \%$ & [151] \\
\hline & & GO & 2 & $\sigma: \uparrow 61.9 \% ; E: \uparrow 104.9 \% ; \varepsilon: \uparrow 223.2 \%$ & [152] \\
\hline \multirow[t]{10}{*}{ PCL } & Solution mixing & PSS-graphene nanosheets (f) & 0.5 & $\sigma: \uparrow 12.8 \% ; E: \uparrow 11.8 \%, \varepsilon: \uparrow 13.2 \%$ & [153] \\
\hline & & GO & 2 & $\sigma: \uparrow 117.4 \% ; E: \uparrow 116.1 \% ; \varepsilon: \uparrow 7.4 \%$ & [154] \\
\hline & & GO nanoplatelets & 0.3 & $\sigma: \uparrow 95 \% ; E: \uparrow 66 \%$ & [155] \\
\hline & & GO & 0.1 & $\sigma: \uparrow 160 \% ; E: \uparrow 103 \%$ & [156] \\
\hline & & Reduced GO & & $\sigma: \uparrow 304 \% ; E: \uparrow 163 \%$ & \\
\hline & & GO (stirred) & 1 & $\sigma: \uparrow 8.3 \% ; E: \uparrow 20.9 \% ; \varepsilon: \downarrow 13.9 \%$ & [157] \\
\hline & & GO (ultrasonicated) & & $\sigma: \uparrow 14.1 \% ; E: \uparrow 37.4 \% ; \varepsilon: \downarrow 7.2 \%$ & \\
\hline & & Graphite (stirred) & & $\sigma: \uparrow 5.1 \% ; E: \uparrow 14.5 \% ; \varepsilon: \downarrow 14.7 \%$ & \\
\hline & & Graphene & 1 & $\sigma: \uparrow 138.4 \% ; E: \uparrow 275 \%$ & [158] \\
\hline & & & 2 & $\sigma: \uparrow 54.1 \% ; E: \uparrow 301.8 \%$ & \\
\hline
\end{tabular}
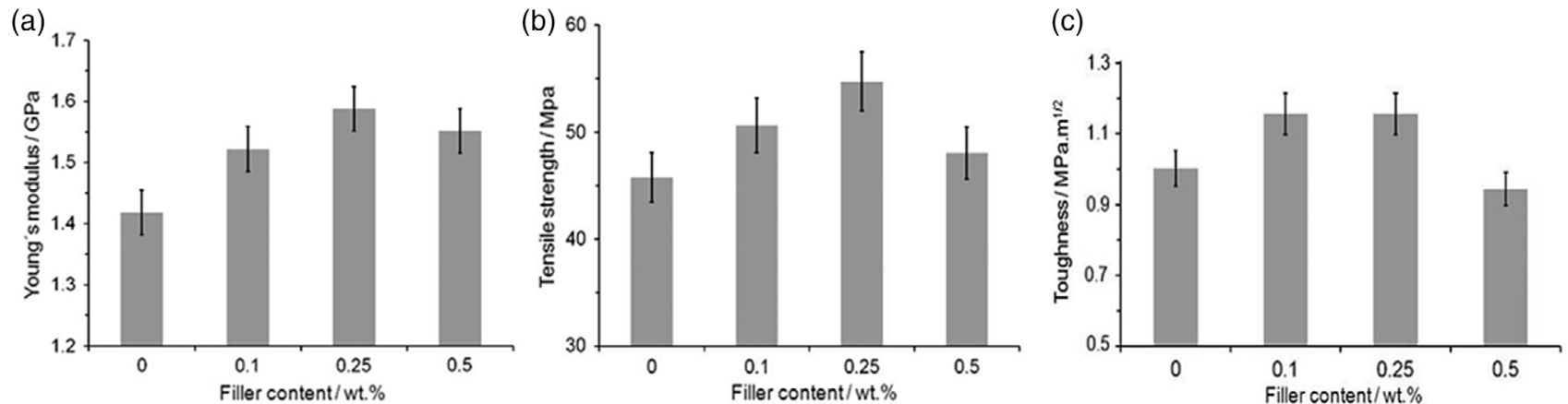

Figure 4. Effect of increasing filler content on mechanical properties of PLA/GNP-C composites a) Young's modulus; b) tensile strength; c) toughness. Reproduced with permission. ${ }^{[147]}$ Copright 2018 , John Wiley and Sons.

reported an increase of $160 \%$ and $103 \%$ in tensile strength and elastic modulus, respectively, for PCL/GO electrospun nanofiber scaffolds, and of 304\% and 163\% for PCL/reduced GO scaffolds, for only $0.1 \mathrm{wt} \%$ loading. The results suggested that smaller loadings were more effective for composite reinforcement, which may be due to good filler dispersion and higher achievable surface area interaction with the molecular chains of the polymer. At higher loadings, the distance between the graphene sheets 
decreased, resulting in a higher tendency to restack, which impeded a uniform filler dispersion and an efficient load transfer, decreasing the efficiency of the mechanical reinforcement effect. These results are in agreement with what is described in the literature.

The properties described show clear dependence on the type, dispersion, and composition of graphene and its derivates. Therefore, careful consideration must be set in the choice of graphene characteristics and composite processing conditions to obtain the best possible reinforcement effect and composite performance. Nevertheless, the presented results are very encouraging, with substantial improvements in mechanical properties being reported, especially at low loadings. It is, thus, demonstrated that graphene may be of great advantage to improve the properties of biodegradable polymers. As such, the resultant composite materials have considerable potential for the development of high-performance scaffold devices with adequate architectures that match appropriately the required mechanical properties and performance for specific tendon/ligament tissues, giving, in this way, an opportunity to these biodegradable polymers to be applied where their use has been restricted. However, improvements in tensile properties are only one step, as other characteristics should also be accessed.

Other Mechanical Properties: Besides the increase in tensile properties, improvements in creep, fatigue, and wear life of graphene-polymer composites have been reported.

Bustillos et al. ${ }^{[160]}$ evaluated the creep behavior of PLAgraphene composites at varying initial loading levels. The authors reported significant improvement in creep resistance of PLAgraphene composites in comparison with the neat polymer. Even at high loading levels, the addition of graphene decreased the creep displacement by $20.5 \%$. Charitos et al. ${ }^{[161]}$ also reported increased creep resistance of PLA/GO (1 wt \%) in comparison with the neat polymer. Similarly, Ramazani et al. ${ }^{[162]}$ also reported an increase in creep resistance of $24 \%$ and $41 \%$ for PCL/GO and PCL/reduced GO composites $(0.1 \mathrm{wt} \%)$, respectively. This enhanced creep resistance can be attributed to the restriction of polymeric chains by graphene, resulting in an effective distribution of load to the graphene reinforcement, preventing further deformation of the polymer network and consequently, resulting in higher resistance to plastic deformation. ${ }^{[160]}$ Similar behavior has been observed in tensile creep testing of other polymer/ graphene composites. ${ }^{[163-165]}$

Increased wear resistance and fatigue life have also been observed with graphene incorporation. Bustillos et al. ${ }^{[160]}$ evaluated the wear resistance of PLA-graphene composites, reporting an increase in $14 \%$ relative to the neat polymer. The authors concluded that graphene's mechanical strength may have contributed to improved wear resistance. ${ }^{[160]}$ Enhanced wear resistance of other graphene-based composites has been reported by other authors. ${ }^{[166-170]}$ Vieira et al. ${ }^{[171]}$ concluded that the addition of GNP (2 wt\%) improves the fatigue behavior of PLA at high strain rates. Bortz et al. ${ }^{[172]}$ observed significant enhancement in fatigue life of GO/epoxy composites $(0.5 \mathrm{wt} \%)$, in comparison with the neat polymer. Mean life at the highest comparable stress level was $420 \%$ greater than that of the control. Improvements at lower stresses were even more remarkable, about $1580 \%$. Other authors have also observed this positive effect in fatigue life. ${ }^{[173-175]}$ It is possible that the addition of graphene may also suppress the formation and expansion of microcracks during cyclic loading, enhancing the lifespan of the composites. ${ }^{[174]}$

Considering the viscoelastic nature of native tendon and ligament tissues, it is important that the scaffold structure presents a similar nonlinear viscoelastic response to loading, which is exhibited by polymer materials, including biodegradable polymers. ${ }^{[171]}$ However, devices for tissue repair are under long-term and repeated loading, so these must also demonstrate good resistance to creep and fatigue, to remain working and provide adequate support during the necessary healing period. The described enhanced creep and wear resistance, along with improvements in the fatigue life of graphene-based polymeric composites, are of extreme importance regarding the desired application. As stated earlier, common problems associated with the use of current commercially available scaffolds include device failure due to fatigue, excessive laxity due to repeated elongation and creep, and lack of durability related to wear. As demonstrated, the application of graphene in biodegradable polymeric scaffolds has the potential to improve their lifetime performance and as such may solve or at least minimize the described problems.

\subsubsection{Effect on Biodegradability}

To be able to use biodegradable polymers in durable applications, it is important to understand the impact of the surrounding conditions on their degradation properties, predict their mean life, as well as improve their resistance to degradation, as even if the initial performance meets the requirements for the target application, their durability in certain conditions may become inadequate for the desired time of use. ${ }^{[176]}$

Biodegradable polyesters are generally degraded by hydrolysis of the ester linkages, a phenomenon that takes place via a diffusion reaction mechanism in which water or other fluids (e.g., body fluids) penetrate the matrix and cause the breakdown of chemical bonds present in the polymer backbone, leading to polymer erosion, which decreases its molecular weight and can even result in the formation of acidic byproducts as previously explained, which can further induce an enhanced water affinity and autocatalyze the reaction, promoting the degradation process. ${ }^{[13,64,98,176]}$ Recently, it has been reported that the inhibition of the diffusion kinetics can be achieved by intercalating graphene in the polymer matrix, which, due to its hydrophobicity, creates a physical barrier that hinders the penetration of water, thus delaying the onset of hydrolysis. ${ }^{[176]}$ Also, as previously stated, the addition of graphene materials may increase the polymer's crystallinity. ${ }^{[176,177]}$ It is well established that the rate of water diffusion can be controlled, in a certain degree, by modifying the material's crystallinity. If the polymer's crystallinity increases, the degradation rate decreases, as it is easier to degrade the amorphous part of the matrix as the higher rate of water uptake occurs in the free volume regions than in the crystalline ones. ${ }^{[63,176,178]}$ Nevertheless, although it has been reported that the particles may act as nucleating agents at low concentrations, at higher concentrations, their interactions with the polymer may affect the crystallinity adversely. ${ }^{[177]}$

Girdthep et al. ${ }^{[179]}$ prepared PLLA/graphene composites by solution mixing and conducted hydrolytic degradation assays 
in sodium hydroxide aqueous solution (initial $\mathrm{pH}=8$ ) at $30^{\circ} \mathrm{C}$. At the end of the study ( 30 days), the authors observed a higher hydrolysis resistance by the PLLA-based composites in the presence of graphene in comparison with the neat sample. Therefore, graphene behaved as a hydrolysis-resistant agent. Furthermore, graphene did not hinder polymer chain mobility and rearrangement during hydrolysis. Pinto et al. ${ }^{[180]}$ incorporated two grades of GNP-M and GNP-C in PLA by melt mixing (0.25 wt\%) and subjected the composites to hydrolytic degradation for 6 months, in phosphate-buffered saline (PBS) at $37^{\circ} \mathrm{C}$, conducting tensile and creep characterization after that period to assess the extent to which the materials were able to maintain their mechanical properties and performance. Although no significant changes were observed in Young's modulus, a decrease in tensile strength, elongation at break, and toughness was higher for PLA (2.6, 2.5, and 10-fold, respectively), compared with PLA/GNP-M (1.6, 1.8, and 3.3 fold) and PLA/GNP-C (1.4, 1.4, and 1.7 fold). Creep results (Figure 5 ) showed that neat PLA presented a higher permanent creep strain in comparison with the non-degraded PLA, whereas both composites displayed behaviors equivalent to the nondegraded samples. Furthermore, after ten creep/ recovery cycles, the degraded neat polymer samples ruptured after four cycles, showing a very intense cumulative increase in permanent creep strain, whereas the composites did not rupture after the tenth cycle, presenting only a slight increase in permanent creep strain. Therefore, the authors concluded that GNP fillers were able to both improve the mechanical properties of PLA and reduced the decay of its mechanical performance during degradation while also being able to significantly counteract the negative effect of biodegradation on PLA's creep stability, attributing this remarkable effect to the fillers' interaction with the matrix.

However, this capacity may be dependent on the type of graphene-based material used. Jeong et al. ${ }^{[181]}$ prepared PLA/GO and PLA/lipophilized-GO functionalized with octadecylamine ( 0.1 and $0.5 \mathrm{wt} \%$ ) composites by solution mixing and conducted hydrolytic degradation studies in a $\mathrm{NaHCO}_{3} / \mathrm{NaOH}$ buffer solution $(\mathrm{pH}=10.7)$, at $60^{\circ} \mathrm{C}$, to analyze the effect of both fillers in the polymer's degradation rate. After 40 days, a high weight loss was verified for neat PLA of $35.8 \%$, of $38.4 \%$ and $48.1 \%$ for PLA/GO, and of $31.4 \%$ and $17.2 \%$ for PLA/functionalized GO, for 0.1 and $0.5 \mathrm{wt} \%$, respectively. According to the authors' remarks, while the hydrophilicity of GO might have accelerated water permeation, the hydrophobic nature of the functionalized filler might have acted as a barrier for water permeation efficiently to reduce the hydrolytic degradation rate of PLA, so portions of the polymer remained protected for a longer period, providing enhanced hydrolytic stability along time. Mohammadi et al. ${ }^{[154]}$ also draw the same conclusions. The authors studied the effect of different GO contents (from 0.5 to $2 \mathrm{wt} \%$ ) on the degradation behavior of electrospun PCL composite nanofibers, in a $\mathrm{NaOH}$ solution at $37^{\circ} \mathrm{C}$. In their degraded state, PCL, PCL/GO 0.5 , 1, and 2 wt $\%$ showed 45\%, 55\%, 57\%, and 69\% mass loss, respectively. Clearly, by increasing GO loading, the degradation rate of the PCL scaffolds was also increased. According to the authors' conclusions, the presence of GO sheets may have increased the density of the hydrophilic groups in the polymer chains and the global hydrophilicity of the structure, which resulted in more water adsorptions and hydration of PCL chains, resulting in faster degradation. These works suggest that the hydrophobicity of the graphene fillers may be an important factor to limit water accessibility and retard the hydrolysis of these polyesters, whereas on the contrary, its hydrophilic counterpart, GO, may induce the opposite effect.

However, other works contradict these findings. Park et al., ${ }^{[151]}$ for example, conducted in vitro degradation studies of PLGA/GO composites with different wt\% in a phosphate buffer ( $\mathrm{pH} 7.4$ ) solution at $37^{\circ} \mathrm{C}$, demonstrating enhanced hydrolysis resistance in PLGA/GO composites in comparison with the neat polymer (Figure 6). The highest weight loss was registered for PLGA and, then decreased with increasing GO content. The authors also noticed decreasing $\mathrm{pH}$ values in the test medium with degradation time (from 7.4 to 3.7) in PLGA polymer, which confirmed the release of acidic materials from the polymer and retention of degradation products in the solution, leading to $\mathrm{pH}$ decrease. The $\mathrm{pH}$ changes were also detected for PLGA/GO composites but lower compared with the pristine polymer, meaning that less acidic byproducts were released into the medium.

Again, filler-type and processing conditions may contribute to the existing variability of results (Figure 7). One factor that is not always considered is the quality of the interface. A better interaction between the polymer and graphene can also contribute to a decrease in the degradation rate. ${ }^{[178]}$ On the contrary, weak interactions can create gaps between the reinforcement and matrix, which facilitate the penetration of water molecules into the material, triggering degradation. ${ }^{[182]}$ Furthermore, recent conclusions point that the hydrophobicity of graphene is thickness (a)

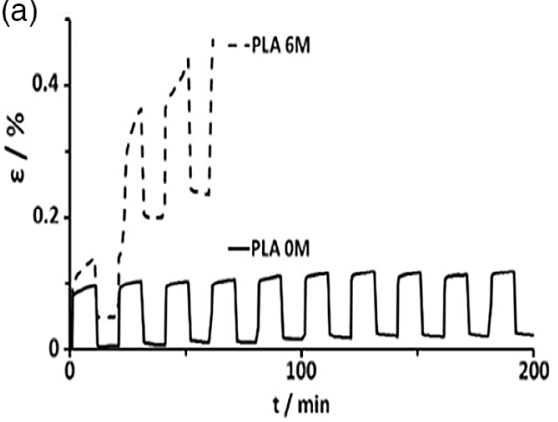

(b)

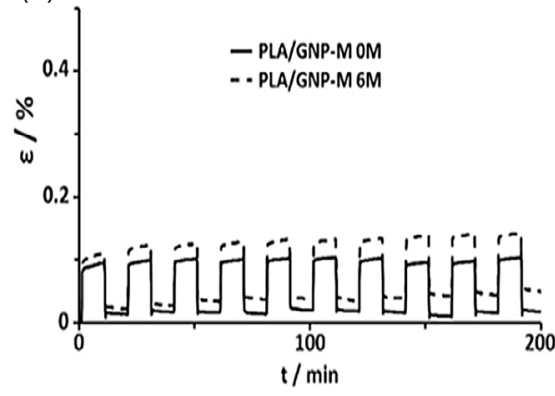

(c)

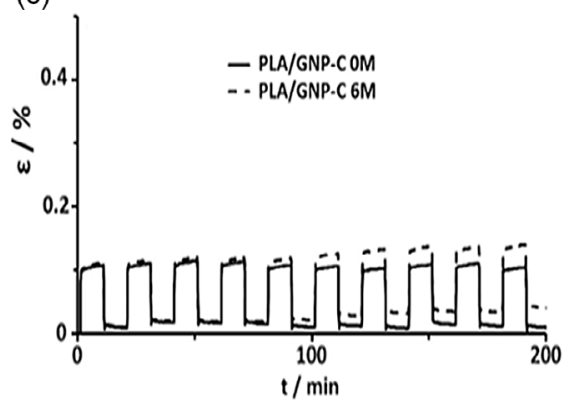

Figure 5. Creep-relaxation curves for a) PLA and b) PLA/GNP-M and c) PLA/GNP-C composites before (0 M) and after 6 months (6 M) degradation. Reproduced with permission. ${ }^{[180]}$ Copyrigyt 2016, Elsevier. 
(a)

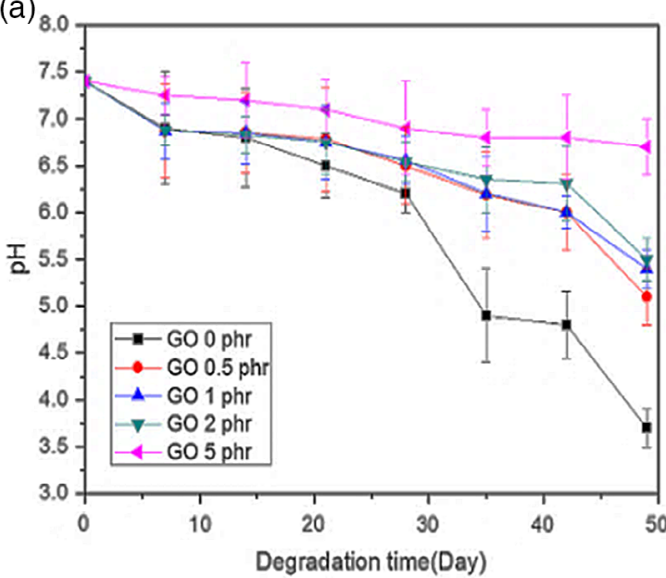

(b)

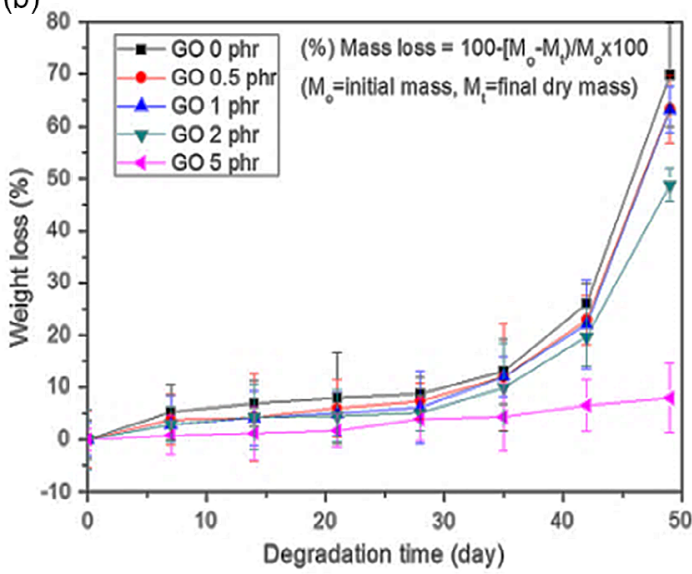

Figure 6. a) $\mathrm{pH}$ changes during degradation of PLGA/GO composites; b) weight loss as a function of hydrolysis time of PLGA/GO composites. Reproduced with permission. ${ }^{[151]}$ Copyrigyt 2014, John Wiley and Sons.

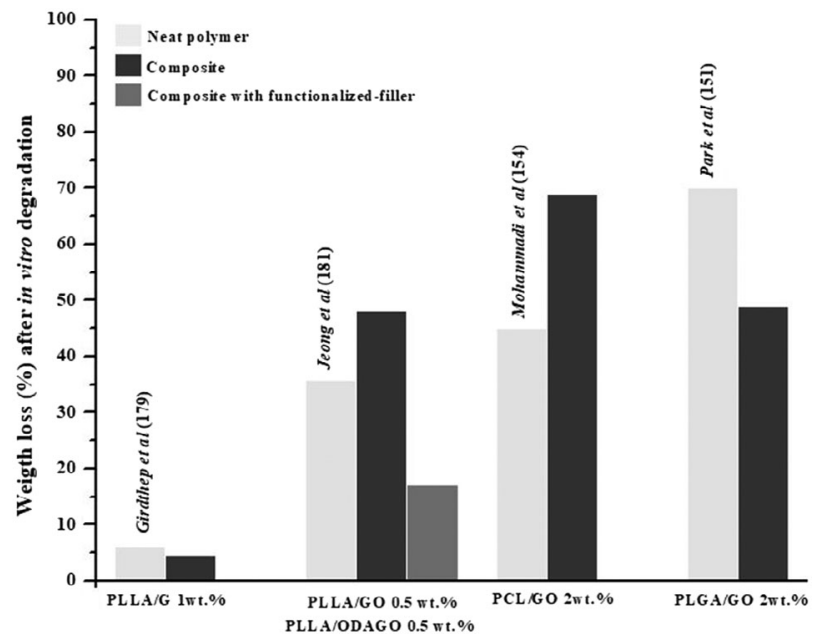

Figure 7. Weight loss (\%) results derived from different in vitro degradation studies of polymer-composite systems.

dependent, with single-layer graphene exhibiting significantly higher hydrophilicity than its thicker counterparts. ${ }^{[183]}$

Degradation properties are of crucial importance in biomaterial selection and design in tendon and ligament repair strategies, as the in vivo degradation behavior can have a crucial impact on the long-term mechanical performance of the structure and on a range of processes such as tissue healing and host response, as previously explained. ${ }^{[25]}$ However, one of the major problems regarding current biodegradable devices is that their mechanical performance cannot be maintained over the necessary healing time, as they begin to degrade and lose strength earlier than desirable. Through the application of graphene materials, it may thus be possible to retard hydrolysis and slow down the degradation rate of these polymers, while increasing and preserving their mechanical properties for longer periods of time. Consequently, the release of acidic byproducts that autocatalyzes the degradation process and causes adverse reactions is also minimized. Furthermore, with this approach, the scaffold performance may be extended without compromising stress transfer to the new forming tissue, as although hydrolytic stability increases, degradation still occurs, although in a minor extent.

\subsubsection{Effect on Biocompatibility}

Although graphene and graphene-based materials are being researched for various biomedical applications, before being considered for use in biomaterials, the in vitro and in vivo evaluation of their biocompatibility and cytotoxicity is of vital importance. ${ }^{[29]}$ However, compared with simpler molecules, where only concentration and exposure type and time are the main toxicological criteria to consider, due to the variability and inhomogeneity of graphene samples, the interactions between these materials with cells, biomolecules, and tissues are various and different and thus require the evaluation of a higher number of factors. ${ }^{[119,136,184]}$ These materials may exhibit dose, size, and shape-dependent cytotoxicity. ${ }^{[10,119,136,185]}$ It is reported that graphene materials do not present in vitro cytotoxicity at a low concentration, exhibiting a low negative influence on cell morphology, viability, and/or proliferation. ${ }^{[110]}$ It is also acknowledged that diverse shapes contribute to differences in cytotoxicity reports. Flat shapes are expected to have minor interactions with cellular membranes and so exhibit less toxicity effects. Indeed, one of the main reasons concerning the application of CNTs in implantable biomedical applications was their tubular shape, which promoted penetration of membranes, resulting in cytotoxicity. ${ }^{[110,186]}$ Third, size may also play an important role on biocompatibility although the reported conclusions are still debatable. Some authors report a higher cellular uptake and toxicity for smaller particles, whereas others report nano-sized graphene materials to be much safer. ${ }^{[110,186,187]}$ Furthermore, the biocompatibility of these materials may also be dependent on their chemical composition. According to literature, functionalized graphene materials present improved biocompatibility by improving hydrophilicity and providing potential cell adhesion sites, like hydroxyl groups. ${ }^{[110,136,185,186]}$ 
Nevertheless, it has been demonstrated that the possibility of a toxic effect of the fillers is even more diminished when incorporated in biomaterials. ${ }^{[184]}$ Graphene-based polymer composites have been investigated as scaffolds for cell culture and tissue engineering and have exhibited negligible harmful effects. Most of available in vitro studies report that graphene and its derivates are biocompatible, do not tend to decrease metabolic activity, and present low cytotoxicity with various cell types, including human mesenchymal stem cells, human osteoblasts, and fibroblasts. ${ }^{[3,121,135]}$ Indeed, many studies have pointed out that graphene and graphene-based materials are excellent substrates for promoting proper cell adhesion, proliferation, and differentiation, as well as enhancing cell activities, in this context. ${ }^{[33,110,135,188]}$

Gonçalves et al. ${ }^{[147]}$ studied the biocompatibility of PLA/GNPs films $(0.25 \mathrm{wt} \%)$ by culturing human foreskin fibroblasts (HFF-1) at the material's surface. The incorporation of GNPs did not affect the cells' metabolic activity or morphology, in comparison with the neat polymer. As such, the incorporation of GNPs had no negative impact on cell growth. The authors concluded that the low filler loading, together with the platelets being well encapsulated in the polymer, were the reasons for the lack of toxicity observed. Pinto et al. went further ${ }^{[180]}$ by evaluating the biocompatibility of the degradation products of PLA/GNP (GNP-M and GNP-C) composites ( $0.25 \mathrm{wt} \%$ ) after 6 months of hydrolytic degradation. When in contact with the degradation products, HFF-1 cells presented low cell death and typical "spindle"-like shapes. So the authors concluded that no toxic products were released in that period. This is relevant considering that long-term biocompatibility must be assured for safe polymer/graphene composite implantation. Pinto et al. ${ }^{[15]}$ also reported PLA/GNP (2 wt\%) to be biocompatible, both in vitro and in vivo. In vitro findings using HFF-1 cells showed that the presence of GNPs in the PLA matrix did not affect the viability or proliferation of seeded cells. The composites registered values above $99 \%$ for cellular metabolic activity (normalized to PLA control), and were able to support cell proliferation, with values up to $136 \%$ being determined in comparison to PLA. In their in vivo study, after 2 weeks of subcutaneous implantation in a mouse model, the implantation site did not reveal any signs of severe inflammation and the granulation tissue of the implant area presented good tissue remodeling, a sign of a healthy healing process.

In two different works, Yoon et al. ${ }^{[152,189]}$ demonstrated enhanced biocompatibility of PLGA/GO composites with two different cell lines, HeLa and neuronal PC 12 cells, due to the addition of GO. Neuronal cells on PLGA/GO (2 wt\%) composite nanofibers [152] exhibited enhanced neurite outgrowth and branching compared with cells in the composites with the lowest GO concentration (1 wt\%) and in the neat PLGA nanofibers. After 2 days of culture, cell proliferation and viability data showed significant differences in metabolic activities, again, being better for PLGA/GO (2 wt\%) compared other conditions. Similar results were obtained for HeLa cells on PLGA/GO composite films. ${ }^{[189]}$ Significant differences in metabolic activities for different samples were found, with better results reported for the composite with the highest GO concentration (2 wt\%). Also, HeLa cells were found adhered and well spread on the surface of PLGA/GO (2 wt\%), illustrating their good affinity with the substrate, with a well-preserved cell membrane, compared with the cells on PLGA and PLGA/GO (1 wt\%). Esrafilzadeh et al. ${ }^{[190]}$ also demonstrated the suitability of PLGA/graphene fibers as a biocompatible, cell-supportive substrate by reporting good attachment, growth, and proliferation of skeletal muscle myoblast (C2C12) cells over the cell culture period. These cells were seen to adhere and elongate along the longitudinal direction of the fibers. Also, cells proliferated to cover the entire fibers' surface within 3 days of culture.

Su et al. ${ }^{[191]}$ also proved the effectiveness of a GO-doped PLGA nanofibrous membrane for the regeneration of tendon to bone enthesis. An in vitro evaluation demonstrated that the composite scaffolds accelerated the proliferation of bone marrow stromal cells and promoted their osteogenic differentiation (Figure 8a). Quantitative analysis revealed that the ALP activity of BMSCs cultured on the PLGA and PLGA/GO membranes was higher than that on the blank control (BC) group, and this increase was higher for the composite (after 7 and 14 days). Furthermore, an in vivo study in a rabbit model further demonstrated that by applying the composite membrane to the gap between a rotator cuff tendon and the bone, the healing of the enthesis was promoted. The PLGA/GO membrane revealed significant capacity to support new bone and cartilage formation at the tendon-bone interface. At 8 and 12 weeks of the study, the control group was filled with fibrous scar tissue and did not present new cartilage regeneration. A few chondrocytes and some new bone were found at the interface in the case of PLGA, whereas a larger new cartilage formation was observed in the PLGA/GO group (Figure 8b). Furthermore, at all time points, the PLGA/GO composite membrane demonstrated the significantly improved production of type I collagen at the healing site in comparison with the other groups, and biomechanical properties were also improved in comparison with the PLGA and control groups, which emphasized the significance of GO during the process of tendon-bone healing.

Murray et al. ${ }^{[192]}$ synthesized PCL/rGO scaffolds by the ringopening polymerization of $\varepsilon$-caprolactone under the exposure of GO nanosheets. After culturing L929 fibroblasts on their surface, the authors observed good cell adhesion and proliferation, forming good focal adhesions, and covering the entire scaffold. The cells grew with normal morphology and cell density increased over the culture period, although at lower rates than the tissue culture control surface $\left(5400 \pm 300\right.$ cells cm$^{-2}$ on PCL and $10000 \pm 2000$ cells cm${ }^{-2}$ on $1 \% \mathrm{PCl} / \mathrm{rGO}$ and $19200 \pm 700$ cells cm ${ }^{-2}$ on the control). Nonetheless, these results illustrate the good biocompatibility of the composite scaffold. Mohammadi et al. ${ }^{[154]}$ performed in vitro MG63 cell culture studies on electrospun PCL/GO nanofibrous scaffolds. The authors reported that the incorporation of GO did not decrease the biocompatibility of PCL nanofibers. On the contrary, cell adhesion and proliferation increased over time. The results showed the highest optical density value in PCL-GO (2 wt\%) group compared with PCL, indicating that cells on fibers with the highest GO concentration had also the highest proliferation rate. The observed cells exhibited the usual spreading morphology with typical nuclei, demonstrating the suitability of the environment for cell organization and colony formation. Moreover, cells cultured on PCL-GO reached more confluency and broadened with large areas, which confirmed that the composites present higher cell attachment. As such, this study indicated that GO 
(a)
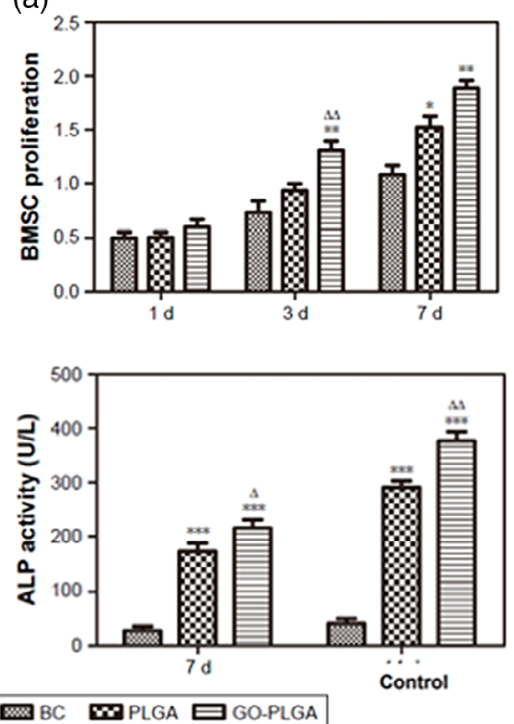

(b)

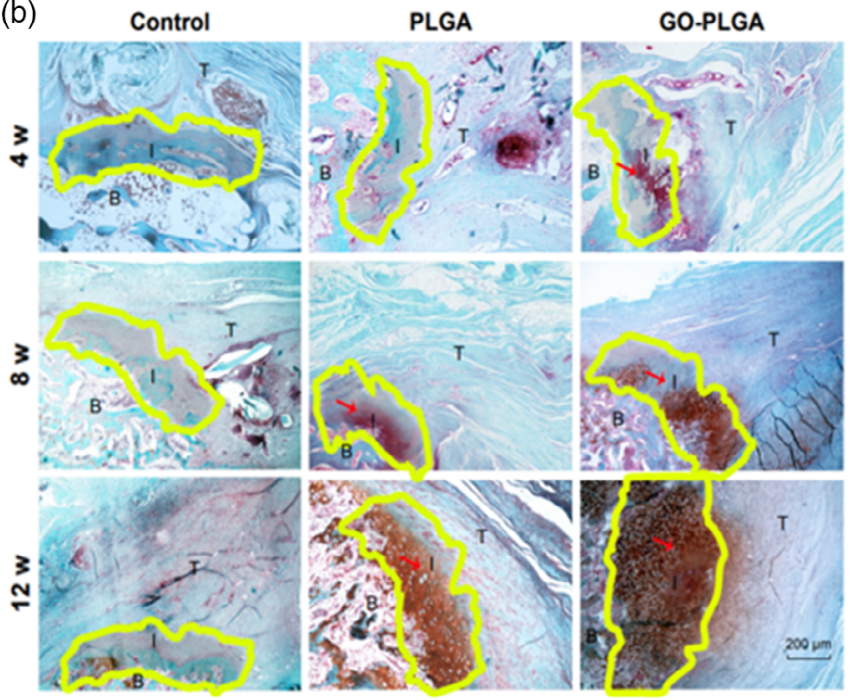

Figure 8. a) Results on proliferation and ALP activity in BC, PLGA, and PLGA/GO groups after BMSCs were seeded onto electrospun nanofibrous membranes. b) Representative histology images of the cartilage tissue at the insertion site. Reproduced with permission. ${ }^{[191]}$ Copyright 2019 , The Authors, published by Dove Medical Press Ltd.

is favorable for the adhesion and proliferation of cells. These positive results have also been reported by other authors, such as Wang et al., ${ }^{[193]}$ that produced PCL/pristine graphene scaffolds for tissue-repair applications, demonstrating that the addition of graphene had a positive impact on human adipose-derived stem cell viability and proliferation, and such as Sayyar et al., ${ }^{[194]}$ which also demonstrated the biocompatibility and suitability of PCL/ graphene scaffolds to support the adhesion and proliferation of different cell lines (L929 fibroblasts, PC-12 neural cells, and C2C12 muscle cells).

The ability to be biocompatible and biofunctional is extremely important to guarantee the success of tissue-repair strategies. The presented polymers are biocompatible and the addition of graphene and graphene-based materials does not change that, and sometimes even improves their biofunctionality, as demonstrated through the previous reported in vitro studies through enhanced adhesion, proliferation, and metabolic activity displayed by several cell lines, including fibroblasts, which are the main intrinsic cell types responsible for the repair of damaged tendon and ligament tissues and also through the enhanced healing reported in in vivo studies. Indeed, in a recent study, Lasocka et al. ${ }^{[195]}$ evaluated the cytotoxicity of the pristine graphene monolayer and its utility as a scaffold for fibroblast L929 cell line. The toxicity degree was assessed based on cell morphology, viability, and mitochondrial activity. Cell motility was evaluated using a wound-healing assay, in which a scratch was made on a precultured cell monolayer, and cell migration was evaluated by analyzing the cells that had migrated into the wound area after a posterior incubation period. Graphene was found to have no cytotoxicity on the studied fibroblasts and increased cell adhesion and proliferation within $24 \mathrm{~h}$ of culture. During the culture period, round-shaped cells gradually changed into a spindle form (Figure 9a). The proliferation of cells grown on graphene was also significantly higher $(113.5 \pm 3.2 \%)$ in comparison with the control group (100 $\pm 3.8 \%$ ) (Figure $9 \mathrm{~b})$.
Furthermore, "wound" closure in the graphene experimental group occurred as a result of linear, regular, and organized cell migration through the pristine scaffold, originating from both edges of the scratch, whereas in the control group (glass substrate), this migration was less organized. After complete closure of the "wound" took place, there were no significant differences in the mean area of fibroblasts migrating into the scratch, although cells on the graphene substrate presented a slightly larger area.

All the earlier findings combined suggested that graphene could be beneficial for damaged tissue recovery, having the potential to support and even improve the healing capacity of damaged tendons and ligaments. Overall, these fillers have shown to not cause cytotoxicity in small studies. As a result, they are being contemplated with optimism in terms of their potential for biomedical applications. However, simple cytotoxicity tests do not satisfy the strict requirements for the translation of novel nanomaterials into clinical use. Long-term in vivo investigations of the biocompatibility of graphene are required to fully establish its biocompatibility and safe use for biomedical applications. ${ }^{[119,136,196]}$

\section{Conclusions}

Despite all the progress observed during the past years regarding the development of a new scaffold for tendon/ligament repair, an ideal one has not been reported yet. Among the several challenges associated with current existent solutions, the main one remains to match the mechanical properties of the native tissues, which is of significant importance for the achievement of a functional repair.

The present Review introduces a novel strategy for tendon and ligament repair, based on the introduction of graphene fillers into biodegradable synthetic polymers as a potential solution to improve their properties and allow their use in such demanding applications. Results available in the literature are very 
(a)

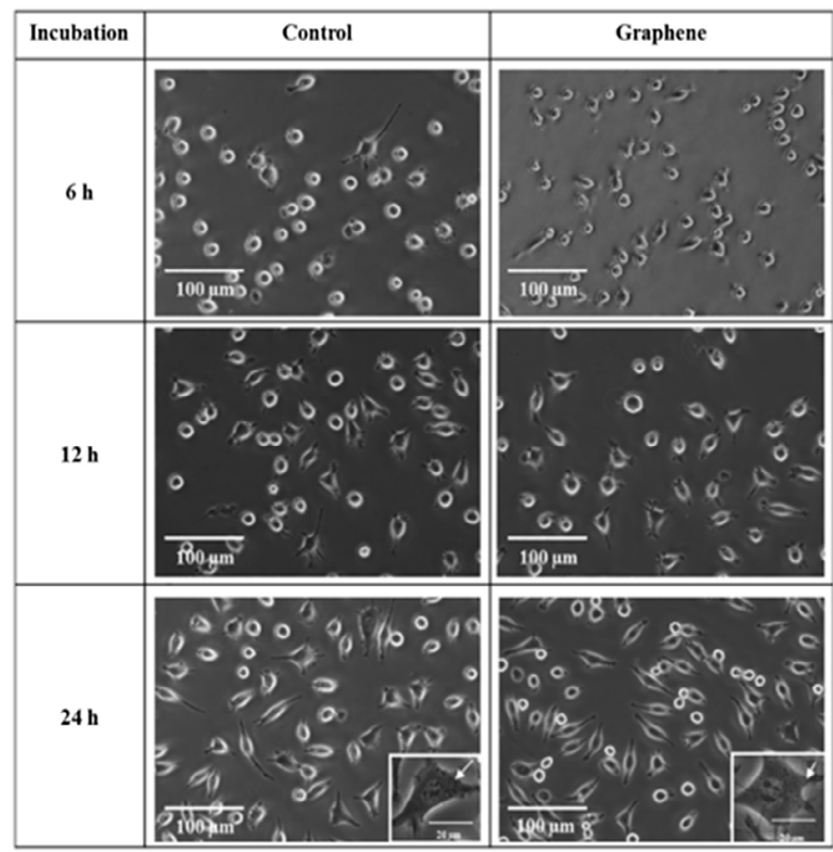

(b)

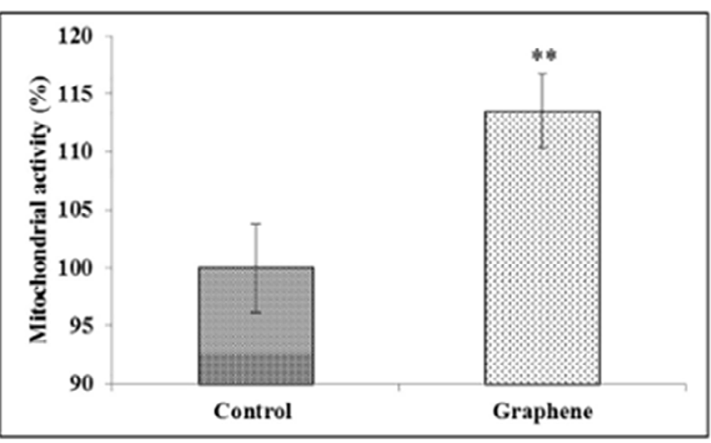

Figure 9. a) Morphology of L929 fibroblasts grown on glass (control) or graphene. b) Mitochondrial activity (proliferation assay) of L929 fibroblasts grown on glass or graphene substrate. Reproduced with permission. ${ }^{[195]}$ Copyright 2018, Elsevier.

encouraging, with substantial improvements in mechanical properties being reported along with their preservation for longer periods of time. Furthermore, graphene-polymer composites have been found to have no cytotoxicity on several cell lines and the introduction of graphene has been proved to be beneficial to enhance the healing rate of tendon and ligament tissues. It can thus be possible to conclude that the addition of graphene fillers can potentially improve the properties of conventional materials and overcome the shortcomings that have been resulting in failure and preventing their use. If an improved biodegradable material is available, a posterior adequate choice in terms of the scaffold structure, namely regarding its final shape, size, architecture, and porosity, can pave the way for the development of innovative structures for tendon and ligament repair, as these factors also influence the properties of the final device. Therefore, these composites present a great potential to overcome the limitations of current devices and finally improve clinical outcomes.

The enormous amount of effort that has been put into graphene research makes it easier to expect with optimism that graphene will, at a future time, transition to an industrial level and become a fundamental part of economic development in several fields, including biomedicine. However, an emergent need of more research in terms of in vivo long-term toxicity is crucial for their use in biomedical applications and transition into the clinic.

\section{Acknowledgments}

IPC acknowledges the support of the Portuguese Foundation for Science and Technology (FCT) through the National Funds References
UIDB/05256/2020 e UIDP/05256/2020. REQUIMTE-LAQV acknowledges the support of the Portuguese Foundation for Science and Technology (FCT) through the National Funds Reference UIDB/50006/2020. Tânia Peixoto also acknowledges the financial support from FCT, through the PhD Grant PD/BD/143035/2018.

\section{Conflict of Interest}

The authors declare no conflict of interest.

\section{Keywords}

biocompatibility and biodegradability, composite scaffolds, graphene, mechanical properties, tendon and ligament repair

Received: April 23, 2020

Revised: July 10, 2020

Published online: September 3, 2020

[1] D. Docheva, S. A. Müller, M. Majewski, C. H. Evans, Adv. Drug Deliv. Rev. 2015, 84, 222.

[2] D. S. Morais, J. Torres, R. M. Guedes, M. A. Lopes, Ann. Biomed. Eng. 2015, 43, 2025.

[3] G. Walden, X. Liao, S. Donell, M. J. Raxworthy, G. P. Riley, A. Saeed, Tissue Eng. Part B Rev. 2017, 23, 44.

[4] A. Ratcliffe, D. L. Butler, N. A. Dyment, P. J. Cagle, C. S. Proctor, S. S. Ratcliffe, E. L. Flatow, Ann Biomed Eng. 2015, 43, 819.

[5] B. B. Rothrauff, B. B. Lauro, G. Yang, R. E. Debski, V. Musahl, R. S. Tuan, Tissue Eng. Part A 2017, 23, 378.

[6] M. V. Hogan, N. Bagayoko, R. James, T. Starnes, A. Katz, B. A. Chhabra, J. Am. Acad. Orthop. Surg. 2011, 19, 134. 
[7] J. Anjana, S. Deepthi, K. T. Shalumon, U. Mony, J.-P. Chen, R. Jayakumar, in Nanoengineered Biomaterials for Regenerative Medicine, Elsevier, Amsterdam 2019, pp. 73-93.

[8] C. Legnani, A. Ventura, C. Terzaghi, E. Borgo, W. Albisetti, Int. Orthop. 2010, 34, 465.

[9] M. T. Rodrigues, R. L. Reis, M. E. Gomes, J. Tissue Eng. Regen. Med. 2013, 7, 673.

[10] S. L. Y. Woo, J. R. Mau, H. Kang, R. Liang, A. J. Almarza, M. B. Fisher, in Principles of Regenerative Medicine, Elsevier, Amsterdam 2019, pp. 1179-1198.

[11] E. T. Ricchetti, A. Aurora, J. P. Iannotti, K. A. Derwin, J. Shoulder Elb. Surg. 2012, 21, 251.

[12] U. D'Amora, A. Gloria, L. Ambrosio, in Biomedical Composites, 2nd ed., Elsevier, Amsterdam 2017, pp. 215-235.

[13] A. C. Vieira, R. M. Guedes, A. T. Marques, J. Biomech. 2009, 42, 2421.

[14] A. L. Kwansa, J. W. Freeman, in Regenerative Engineering of Musculoskeletal Tissues and Interfaces, (Eds.: S. P. Nukavarapu, J. W. Freeman, C. Laurencin), Woodhead Publishing, Amsterdam 2015, pp. 161-193.

[15] V. Correia Pinto, R. Costa-Almeida, I. Rodrigues, L. Guardão, R. Soares, R. Miranda Guedes, J. Biomed. Mater. Res. Part A 2017, 105, 2182.

[16] F. Alshomer, C. Chaves, D. M. Kalaskar, J Mater. 2018, 2018, 9868151.

[17] J. C.-H. Goh, H.-W. Ouyang, S.-H. Teoh, C. K. C. Chan, E.-H. Lee, Tissue Eng. 2003, 9, 31.

[18] M. R. Sanjay, G. R. Arpitha, B. Yogesha, Mater. Today Proc. 2015, 2, 2959.

[19] H. Cheung, M. Ho, K. Lau, F. Cardona, D. Hui, Compos. Part B Eng. 2009, 40, 655.

[20] J. K. Carrow, A. K. Gaharwar, Macromol. Chem. Phys. 2015, 216, 248.

[21] M. Bhattacharya, Materials 2016, 9, 262.

[22] A. K. Gaharwar, N. A. Peppas, A. Khademhosseini, Biotechnol. Bioeng. 2014, 111, 441.

[23] J.-M. Raquez, Y. Habibi, M. Murariu, P. Dubois, Prog. Polym. Sci. 2013, 38, 1504

[24] R. Urie, D. Ghosh, I. Ridha, K. Rege, Annu. Rev. Biomed. Eng. 2018, 20, 353.

[25] I. Armentano, M. Dottori, E. Fortunati, S. Mattioli, J. M. Kenny, Polym. Degrad. Stab. 2010, 95, 2126.

[26] B. Sharma, P. Malik, P. Jain, Mater Today Commun. 2018, 16, 353.

[27] S. Ganguly, P. Das, S. Banerjee, N. C. Das, Funct. Compos. Struct. 2019, 1, 22001

[28] V. B. Mohan, K. Lau, D. Hui, D. Bhattacharyya, Compos. Part B Eng. 2018, 142, 200.

[29] C. Gonçalves, I. C. Gonçalves, F. D. Magalhães, A. M. Pinto, Polymers 2017, 9, 269.

[30] A. Zhang, X. Ji, J. Liu, in Carbon-Based Polymer Nanocomposites for Environmental and Energy Applications, Elsevier, Amsterdam 2018, pp. 147-173.

[31] D. G. Papageorgiou, I. A. Kinloch, R. J. Young, Prog. Mater. Sci. 2017, 90, 75.

[32] S. R. Shin, Y.-C. Li, H. L. Jang, P. Khoshakhlagh, M. Akbari, A. Nasajpour, Y. S. Zhang, A. Tamayol, A. Khademhosseini, Adv. Drug Deliv. Rev. 2016, 105, 255.

[33] Y. Zhang, T. R. Nayak, H. Hong, W. Cai, Nanoscale 2012, 4, 3833.

[34] A. Sensini, L. Cristofolini, Materials 2018, 11, 1963.

[35] P. S. Thayer, A. S. Goldstein, in Bio-Instructive Scaffolds for Musculoskeletal Tissue Engineering and Regenerative Medicine, Elsevier, Amsterdam 2017, pp. 87-112.

[36] H. Asahara, M. Inui, M. K. Lotz, J. Bone Miner. Res. 2017, 32, 1773.

[37] W. L. Lim, L. L. Liau, M. H. Ng, S. R. Chowdhury, J. X. Law, Tissue Eng. Regen. Med. 2019, 16, 549.
[38] Y. J. No, M. Castilho, Y. Ramaswamy, H. Zreiqat, Adv Mater. 2020 32, 1904511.

[39] M. Lavagnino, M. E. Wall, D. Little, A. J. Banes, F. Guilak, S. P. Arnoczky, J. Orthop. Res. 2015, 33, 813.

[40] M. L. Santos, M. T. Rodrigues, R. M. A. Domingues, R. L. Reis, M. E. Gomes, in Regenerative Strategies for the Treatment of Knee Joint Disabilities, Springer, Cham 2017, pp. 349-371.

[41] N. L. Leong, J. L. Kator, T. L. Clemens, A. James, M. EnamotoIwamoto, J. Jiang, J. Orthop. Res. 2020, 38, 7.

[42] C. B. Frank, J. Musculoskelet. Neuronal Interact. 2004, 4, 199.

[43] R. B. Martin, D. B. Burr, N. A. Sharkey, D. P. Fyhrie, in Skeletal Tissue Mechanics, Springer, New York 2015, pp. 175-225.

[44] P. Sharma, N. Maffulli, J. Bone Joint Surg. Am. 2005, 87, 187.

[45] D. Ö. Kaya, in Comparative Kinesiology of the Human Body. Elsevier, Amsterdam 2020, pp. 115-147.

[46] S. P. Reese, J. A. Weiss, in Multiscale Modeling in Biomechanics and Mechanobiology, Springer, London 2015, pp. 159-206.

[47] U. G. Longo, M. Ronga, N. Maffulli, Sports Med. Arthrosc. 2018, 26, 16.

[48] S. Thomopoulos, W. C. Parks, D. B. Rifkin, K. A. Derwin, J. Orthop. Res. 2015, 33, 832

[49] B. A. Stewart, A. M. Momaya, M. D. Silverstein, D. Lintner, Am. J. Sports Med. 2017, 45, 23.

[50] J. A. Cottrell, J. C. Turner, T. L. Arinzeh, J. P. O'Connor, Foot Ankle Clin. 2016, 21, 739.

[51] C. N. Riggin, T. R. Morris, L. J. Soslowsky, in Tendon Regeneration, Elsevier, London 2015, pp. 149-183.

[52] C. S. Chamberlain, E. E. Saether, E. Aktas, R. Vanderby, J. Cytokine Biol. 2017, 2, 112.

[53] E. Naghashzargar, S. Farè, V. Catto, S. Bertoldi, D. Semnani, S. Karbasi, M. C. Tanzi, J. Appl. Biomater. Funct. Mater. 2015, 13, 156

[54] C. T. Laurencin, J. W. Freeman, Biomaterials 2005, 26, 7530.

[55] U. G. Longo, A. Lamberti, N. Maffulli, V. Denaro, Br. Med. Bull. 2010, 94, 165.

[56] J. Chen, J. Xu, A. Wang, M. Zheng, Expert Rev. Med. Devices 2009 6,61 .

[57] S. F. Badylak, D. O. Freytes, T. W. Gilbert, Acta Biomater. 2009, 5, 1.

[58] F.-M. Chen, X. Liu, Prog. Polym. Sci. 2016, 53, 86.

[59] A. Asti, L. Gioglio, Int. J. Artif. Organs 2014, 37, 187.

[60] S. Doppalapudi, A. Jain, W. Khan, A. J. Domb, Polym. Adv. Technol. 2014, 25, 427.

[61] R. Suntornnond, J. An, W. Y. Yeong, C. K. Chua, Macromol. Mater. Eng. 2015, 300, 858.

[62] M. T. Wolf, C. L. Dearth, S. B. Sonnenberg, E. G. Loboa, S. F. Badylak, Adv Drug Deliv Rev. 2015, 84, 208.

[63] M. S. Cortizo, M. S. Belluzo, in Industrial Applications of Renewable Biomass Products, Springer, Cham 2017, pp. 47-74.

[64] N. Iqbal, A. S. Khan, A. Asif, M. Yar, J. W. Haycock, I. U. Rehman, Int Mater. Rev. 2019, 64, 91.

[65] I. Manavitehrani, A. Fathi, H. Badr, S. Daly, A. Negahi Shirazi, F. Dehghani, Polymers 2016, 8, 20.

[66] J. A. Cooper, H. H. Lu, F. K. Ko, J. W. Freeman, C. T. Laurencin, Biomaterials. 2005, 26(13), 1523.

[67] Synthasome, X-repair product [Internet], http://www.synthasome. com/xRepair.php (accessed: December 2018).

[68] G. R. Waryasz, S. Marcaccio, J. A. Gil, B. D. Owens, P. D. Fadale, JBJS Rev. 2017, 5, e2.

[69] Y. Wu, Y. Han, Y. S. Wong, J. Y. H. Fuh, J. Tissue Eng. Regen. Med. 2018, 12, 1798

[70] C. Laurent, X. Liu, N. De Isla, X. Wang, R. Rahouadj, J. Cell Immunother. 2018, 4, 4.

[71] A. Chainani, D. Little, Tech. Orthop. 2016, 31, 91.

[72] M. L. Killian, L. Cavinatto, L. M. Galatz, S. Thomopoulos, J. Shoulder Elb. Surg. 2012, 21, 228. 
[73] C. Z. Liu, J. T. Czernuszka, Mater. Sci. Technol. 2007, 23, 379.

[74] C. K. Kuo, J. E. Marturano, R. S. Tuan, BMC Sports Sci. Med. Rehabil. 2010, 2, 20.

[75] J. C. Middleton, A. J. Tipton, Biomaterials 2000, 21, 2335.

[76] F. Wahid, T. Khan, Z. Hussain, H. Ullah, in Applications of Nanocomposite Materials in Drug Delivery, Elsevier, San Diego, CA 2018, pp. 701-735.

[77] S. P. Lake, Q. Liu, M. Xing, L. E. lannucci, Z. Wang, C. Zhao, in Principles of Tissue Engineering, Elsevier, Amsterdam 2020, pp. 989-1005.

[78] M. F. Maitz, Biosurf. Biotribol. 2015, 1, 161.

[79] H. H. Lu, J. A. Cooper Jr., S. Manuel, J. W. Freeman, M. A. Attawia, F. K. Ko, C. T. Laurencin, Biomaterials 2005, 26, 4805.

[80] D. Deng, W. Wang, B. Wang, P. Zhang, G. Zhou, W. J. Zhang, Y. Cao, W. Liu, Biomaterials 2014, 35, 8801.

[81] O. Laitinen, P. Törmälä, R. Taurio, K. Skutnabb, K. Saarelainen, T. livonen, S. Vainionpää, Biomaterials 1992, 13, 1012.

[82] J. A. Cooper, J. S. Sahota, W. J. Gorum, J. Carter, S. B. Doty, C. T. Laurencin, Proc. Natl. Acad. Sci. 2007, 104, 3049.

[83] M. C. Araque-Monrós, T. C. Gamboa-Martínez, L. G. Santos, S. G. Bernabé, M. M. Pradas, J. M. Estellés, J. Biomed. Mater. Res. Part A 2013, 101, 3228.

[84] K. L. Moffat, A. S.-P. Kwei, J. P. Spalazzi, S. B. Doty, W. N. Levine, H. H. Lu, Tissue Eng. Part A 2008, 15, 115.

[85] H. W. Ouyang, J. C. H. Goh, A. Thambyah, S. H. Teoh, E. H. Lee, Tissue Eng. 2003, 9, 431.

[86] N. L. Leong, N. Kabir, A. Arshi, A. Nazemi, B. Wu, F. A. Petrigliano, D. R. McAllister, Tissue Eng. Part A 2015, 21, 1859.

[87] Y. Wu, Z. Wang, J. Y. H. Fuh, Y. San Wong, W. Wang, E. San Thian, J. Mater. Sci. Mater. Med. 2016, 27, 115.

[88] M.-T. Cheng, Y.-R. V.V. Shih, O. K. Lee, in Stem Cell Biology and Tissue Engineering in Dental Sciences, Elsevier, Amsterdam 2015, pp. 553-565.

[89] M. A. Velasco, C. A. Narváez-Tovar, D. A. Garzón-Alvarado, Biomed. Res Int. 2015, 2015, 729076.

[90] Z. Sheikh, S. Najeeb, Z. Khurshid, V. Verma, H. Rashid, M. Glogauer, Materials 2015, 8, 5744.

[91] S. V. Gohil, S. Suhail, J. Rose, T. Vella, L. S. Nair, in Materials for Bone Disorders, Elsevier, Amsterdam 2017, pp. 349-403.

[92] A. N. Shirazi, W. Chrzanowski, A. Khademhosseini, F. Dehghani, in Engineering Mineralized and Load Bearing Tissues, Springer, Cham 2015, pp. 161-186.

[93] F. Serack, N. Holwell, B. G. Amsden, in Functional Biopolymers (Eds: M. A. J. Mazumder, H. Sheardown, A. Al-Ahmed), Springer, Cham 2018, pp. 347-376.

[94] A. Oluwadamilola, S. Yousaf, M. Zare, M. Mozafari, M. Youseffi, P. Twigg, F. Sefat, in Handbook of Tissue Engineering Scaffolds, vol. 1, Elsevier, Amsterdam 2019, pp. 299-327.

[95] V. C. Pinto, T. Ramos, A. S. F. Alves, J. Xavier, P. J. Tavares, P. Moreira, R. M. Guedesab, Eng. Fail. Anal. 2017, 71, 63.

[96] G. Verdiyeva, K. Koshy, N. Glibbery, H. Mann, A. M. Seifalian, J. Biomed. Nanotechnol. 2015, 11, 1495.

[97] U. G. Longo, A. Lamberti, S. Petrillo, N. Maffulli, V. Denaro, Stem Cells Int. 2012, 2012, 517165.

[98] M. Dziadek, E. Stodolak-Zych, K. Cholewa-Kowalska, Mater. Sci. Eng. C 2017, 71, 1175.

[99] R. A. Hule, D. J. Pochan, MRS Bull. 2007, 32, 354.

[100] S. Ramakrishna, J. Mayer, E. Wintermantel, K. W. Leong, Compos. Sci. Technol. 2001, 61, 1189.

[101] L. M. Cross, A. Thakur, N. A. Jalili, M. Detamore, A. K. Gaharwar, Acta Biomater. 2016, 42, 2.

[102] M. Balasubramanian, in Fibrous and Textile Materials for Composite Applications, Springer, Singapore 2016, pp. 1-38.
[103] R. M. Jones, Mechanics of Composite Materials, CRC Press, New York 2014, pp. 2-11.

[104] F. C. Campbell, Structural Composite Materials, ASM International, Materials Park, $\mathrm{OH}$ 2010, pp. 1-18.

[105] B. Zhang, Y. Wang, G. Zhai, Mater. Sci. Eng. C 2016, 61, 953.

[106] D. Chimene, D. L. Alge, A. K. Gaharwar, Adv. Mater. 2015, 27, 7261.

[107] X. Ji, Y. Xu, W. Zhang, L. Cui, J. Liu, Compos. Part A Appl. Sci. Manuf. 2016, 87, 29.

[108] G. Mittal, V. Dhand, K. Y. Rhee, S.-J. Park, W. R. Lee, J. Ind. Eng. Chem. 2015, 21, 11.

[109] P. Cataldi, A. Athanassiou, I. Bayer, Appl. Sci. 2018, 8, 1438.

[110] X. Cheng, Q. Wan, X. Pei, Nanoscale Res. Lett. 2018, 13, 289.

[111] N. A. A. Ghany, S. A. Elsherif, H. T. Handal, Surf. Interfaces 2017, 9, 93.

[112] Y. Zhong, Z. Zhen, H. Zhu, FlatChem 2017, 4, 20.

[113] Y. Yang, A. M. Asiri, Z. Tang, D. Du, Y. Lin, Mater. Today 2013, 16, 365.

[114] M. E. Foo, S. C. B. Gopinath, Biomed. Pharmacother. 2017, 94, 354.

[115] S. Gurunathan, J.-H. Kim, Int. J. Nanomed. 2016, 11, 1927.

[116] J. Phiri, P. Gane, T. C. Maloney, Mater. Sci. Eng. B 2017, 215, 9.

[117] D. W. Johnson, B. P. Dobson, K. S. Coleman, Curr. Opin. Colloid Interface Sci. 2015, 20, 367.

[118] L.-Z. Guan, L. Zhao, Y.-J. Wan, L.-C. Tang, Nanoscale 2018, 10, 14788.

[119] G. Reina, J. M. González-Domínguez, A. Criado, E. Vázquez, A. Bianco, M. Prato, Chem. Soc. Rev. 2017, 46, 4400.

[120] S. P. Lonkar, Y. S. Deshmukh, A. A. Abdala, Nano Res. 2015, 8, 1039.

[121] S. Pattnaik, K. Swain, Z. Lin, J. Mater. Chem. B 2016, 4, 7813.

[122] E. Cunha, M. Proença, M. Pereira, M. Fernandes, R. Young, K. Strutyński, M. Melle-Franco, M. Gonzalez-Debs, P. E. Lopes, M. da Conceição Paiva, Nanomaterials 2018, 8, 675.

[123] A. Amiri, M. Naraghi, G. Ahmadi, M. Soleymaniha, M. Shanbedi, FlatChem 2018, 8, 40.

[124] V. C. Sanchez, A. Jachak, R. H. Hurt, A. B. Kane, Chem. Res. Toxicol. 2011, 25, 15.

[125] A. Idowu, B. Boesl, A. Agarwal, Carbon 2018, 135, 52.

[126] H. Shen, L. Zhang, M. Liu, Z. Zhang, Theranostics 2012, 2, 283.

[127] T. K. Das, S. Prusty, Polym. Plast. Technol. Eng. 2013, 52, 319.

[128] M. Zhang, Y. Li, Z. Su, G. Wei, Polym. Chem. 2015, 6, 6107.

[129] K. Hu, D. D. Kulkarni, I. Choi, V. V. Tsukruk, Prog. Polym. Sci. 2014, 39, 1934.

[130] L. S. Schadler, L. C. Brinson, W. G. Sawyer, J. Med. 2007, 59, 53.

[131] N. Saravanan, R. Rajasekar, S. Mahalakshmi, T. P. Sathishkumar, K. S. K. Sasikumar, S. Sahoo, J. Reinf. Plast. Compos. 2014, 33, 1158.

[132] J. Bramhill, S. Ross, G. Ross, Int. J. Environ. Res. Public Health 2017, $14,66$.

[133] S. N. Tripathi, G. S. S. Rao, A. B. Mathur, R. Jasra, RSC Adv. 2017, 7, 23615

[134] B. Sreenivasulu, B. R. Ramji, M. Nagaral, Mater Today Proc. 2018, 5, 2419.

[135] A. N. Banerjee, Interface Focus 2018, 8, 20170056.

[136] H. Zhao, R. Ding, X. Zhao, Y. Li, L. Qu, H. Pei, L. Yildirimer, Z. Wu, W. Zhang, Drug Discov. Today 2017, 22, 1302.

[137] S. Ganguly, D. Ray, P. Das, P. P. Maity, S. Mondal, V. K. Aswal, S. Dhara, N. Ch. Das, Ultrason Sonochem. 2018, 42, 212.

[138] S. Ganguly, P. Das, P. P. Maity, S. Mondal, S. Ghosh, S. Dhara, N. Ch. Das, J. Phys. Chem. B 2018, 122, 7201.

[139] D. Wu, Y. Cheng, S. Feng, Z. Yao, M. Zhang, Ind. Eng. Chem. Res. 2013, 52, 6731.

[140] Y. Cao, J. Feng, P. Wu, Carbon 2010, 48, 3834.

[141] X. Li, Y. Xiao, A. Bergeret, M. Longerey, J. Che, Polym. Compos. 2014, 35, 396.

[142] A. M. Pinto, J. Cabral, D. A. P. Tanaka, A. M. Mendes, F. D. Magalhães, Polym. Int. 2013, 62, 33. 
[143] W. Li, Z. Xu, L. Chen, M. Shan, X. Tian, C. Yang, H. Lv, X. Qian, Chem. Eng. J. 2014, 237, 291.

[144] X. Zhang, B. Geng, H. Chen, Y. Chen, Y. Wang, L. Zhang, H. Liu, H. Yang, J. Chen, Chem. Eng. J 2018, 334, 2014.

[145] G. Chakraborty, A. Gupta, G. Pugazhenthi, V. Katiyar, J. Appl. Polym. Sci. 2018, 135, 46476.

[146] C. Bao, L. Song, W. Xing, B. Yuan, C. A. Wilkie, J. Huang, Y. Guo, Y. Hu, J. Mater. Chem. 2012, 22, 6088.

[147] C. Gonçalves, A. Pinto, A. V. Machado, J. Moreira, I. C. Gonçalves, F. Magalhães, Polym. Compos. 2018, 39, E308.

[148] R. Scaffaro, L. Botta, A. Maio, G. Gallo, Compos. Part B Eng. 2017, 109, 138.

[149] E. Narimissa, R. K. Gupta, H. J. Choi, N. Kao, M. Jollands, Polym. Compos. 2012, 33, 1505.

[150] Y. Gao, O. T. Picot, E. Bilotti, T. Peijs, Eur. Polym. J. 2017, 86, 117.

[151] J. J. Park, E. J. Yu, W. Lee, C. Ha, Polym. Adv. Technol. 2014, 25, 48.

[152] O. J. Yoon, C. Y. Jung, I. Y. Sohn, H. J. Kim, B. Hong, M. S. Jhon, N.-E. Lee, Compos. Part A Appl. Sci. Manuf. 2011, 42, 1978.

[153] M. Wang, X.-Y. Deng, A.-K. Du, T.-H. Zhao, J.-B. Zeng, RSC Adv. 2015, 5, 73146.

[154] S. Mohammadi, S. S. Shafiei, M. Asadi-Eydivand, M. Ardeshir, M. Solati-Hashin, J. Bioact. Compat. Polym. 2017, 32, 325.

[155] C. Wan, B. Chen, Biomed. Mater. 2011, 6, 55010.

[156] S. Ramazani, M. Karimi, Mater. Sci. Eng. C 2015, 56, 325.

[157] W. Kai, Y. Hirota, L. Hua, Y. Inoue, J. Appl. Polym. Sci. 2008, 107, 1395.

[158] P. Ginestra, J. Mech. Behav. Biomed. Mater. 2019, 100, 103387.

[159] X.-Z. Tong, F. Song, M.-Q. Li, X.-L. Wang, I.-J. Chin, Y.-Z. Wang, Compos. Sci. Technol. 2013, 88, 33.

[160] J. Bustillos, D. Montero, P. Nautiyal, A. Loganathan, B. Boesl, A. Agarwal, Polym. Compos. 2017, 39, 3877.

[161] I. Charitos, D. Mouzakis, E. Kontou, Polym. Eng. Sci. 2019, 59, 1933.

[162] S. Ramazani, M. Karimi, J. Mech. Behav. Biomed. Mater. 2016, 61, 484.

[163] L.-C. Tang, X. Wang, L.-X. Gong, K. Peng, L. Zhao, Q. Chen, L.-B. Wu, J.-X. Jiang, G.-Q. Lai, Compos. Sci. Technol. 2014, 91, 63.

[164] A. Bhattacharyya, S. Chen, M. Zhu, Express Polym. Lett. 2014, 8, 74.

[165] A. Zandiatashbar, C. R. Picu, N. Koratkar, Small 2012, 8, 1676.

[166] Z. Tai, Y. Chen, Y. An, X. Yan, Q. Xue, Tribol Lett. 2012, 46, 55.

[167] X.-J. Shen, X.-Q. Pei, S.-Y. Fu, K. Friedrich, Polymer 2013, 54, 1234.

[168] S. S. Kandanur, M. A. Rafiee, F. Yavari, M. Schrameyer, Z.-Z. Yu, T. A. Blanchet, N. Koratkar, Carbon 2012, 50, 3178.

[169] D. Cai, J. Jin, K. Yusoh, R. Rafiq, M. Song, Compos. Sci. Technol. 2012, 72, 702.

[170] B. Pan, G. Xu, B. Zhang, X. Ma, H. Li, Y. Zhang, Polym. Plast. Technol. Eng. 2012, 51, 1163.

[171] A. Vieira, V. C. Pinto, A. Pinto, F. D. Magalhães, Int. J. Automot. Compos. 2015, 1, 244.

[172] D. R. Bortz, E. G. Heras, I. Martin-Gullon, Macromolecules 2011, 45, 238.
[173] M. A. Rafiee, J. Rafiee, I. Srivastava, Z. Wang, H. Song, Z. Yu, N. Koratkar, Small 2010, 6, 179.

[174] M.-Y. Shen, T.-Y. Chang, T.-H. Hsieh, Y.-L. Li, C.-L. Chiang, H. Yang, M.-C. Yip, J. Nanomater. 2013, 2013, 1.

[175] F. Yavari, M. A. Rafiee, J. Rafiee, Z.-Z. Yu, N. Koratkar, ACS Appl. Mater. Interfaces 2010, 2, 2738.

[176] A. Porfyris, S. Vasilakos, C. Zotiadis, C. Papaspyrides, K. Moser L. Van der Schueren, G. Buyle, S. Pavlidou, S. Vouyiouka, Polym. Test 2018, 68, 315.

[177] S. Kashi, R. K. Gupta, N. Kao, S. A. Hadigheh, S. N. Bhattacharya, J. Mater. Sci. Technol. 2018, 34, 1026.

[178] M.-X. Li, S.-H. Kim, S.-W. Choi, K. Goda, W.-I. Lee, Compos. Part B Eng. 2016, 96, 248.

[179] S. Girdthep, W. Sankong, A. Pongmalee, T. Saelee, W. Punyodom, P. Meepowpan, P. Worajittiphon, Polym. Test 2017, 61, 229.

[180] A. M. Pinto, C. Gonçalves, I. C. Gonçalves, F. D. Magalhães, Eur. Polym J. 2016, 85, 431.

[181] J. Jeong, M. Choi, S. Nagappan, W. Lee, C. Ha, Polym. Int. 2018 67, 91.

[182] T.-X. Jin, C. Liu, M. Zhou, S. Chai, F. Chen, Q. Fu, Compos. Part A Appl. Sci. Manuf. 2015, 68, 193.

[183] M. Munz, C. E. Giusca, R. L. Myers-Ward, D. K. Gaskill, O. Kazakova, ACS Nano 2015, 9, 8401.

[184] A. M. Pinto, I. C. Goncalves, F. D. Magalhaes, Colloids Surf. B Biointerfaces 2013, 111, 188.

[185] T. P. D. Shareena, D. McShan, A. K. Dasmahapatra, P. B. Tchounwou, Nano-Micro Lett. 2018, 10, 53.

[186] X. Hu, Q. Zhou, Chem Rev. 2013, 113, 3815.

[187] X. Wu, S.-J. Ding, K. Lin, J. Su, J. Mater. Chem. B 2017, 5, 3084

[188] H. Xie, T. Cao, F. J. Rodríguez-Lozano, E. K. Luong-Van, V. Rosa, Dent Mater. 2017, 33, 765.

[189] O. J. Yoon, I. Y. Sohn, D. J. Kim, N.-E. Lee, Macromol Res. 2012, 20, 789.

[190] D. Esrafilzadeh, R. Jalili, E. M. Stewart, S. H. Aboutalebi, J. M. Razal, S. E. Moulton, G. G. Wallace, Adv. Funct. Mater. 2016, 26, 3105.

[191] W. Su, Z. Wang, J. Jiang, X. Liu, J. Zhao, Z. Zhang, Int. J. Nanomed. 2019, 14, 1835.

[192] E. Murray, S. Sayyar, B. C. Thompson, R. Gorkin III, D. L. Officer, G. G. Wallace, RSC Adv. 2015, 5, 45284.

[193] W. Wang, G. Caetano, W. S. Ambler, J. J. Blaker, M. A. Frade, P. Mandal, C. Diver, P. Bártolo, Materials 2016, 9, 992.

[194] S. Sayyar, E. Murray, B. C. Thompson, S. Gambhir, D. L. Officer, G. G. Wallace, Carbon 2013, 52, 296.

[195] I. Lasocka, L. Szulc-Dąbrowska, M. Skibniewski, E. Skibniewska, W. Strupinski, I. Pasternak, H. Kmieć, P. Kowalczyk, Toxicol. Vitr. 2018, 48, 276.

[196] M. Mehrali, A. Thakur, C. P. Pennisi, S. Talebian, A. Arpanaei, M. Nikkhah, A. Dolatshahi-Pirouz, Adv. Mater. 2017, 29, 1603612.

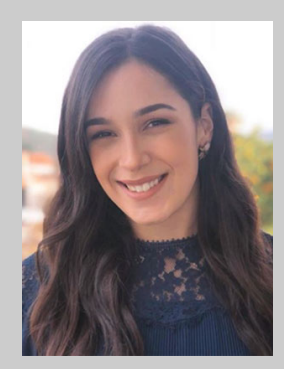

Tânia Peixoto finished her master's degree in Biomedical Engineering at University of Trás-Os-Montes e Alto Douro in 2017, specializing in Bioprocesses and Biomaterials. She is a Ph.D. student in the Doctoral Program in Advanced Materials and Processing at Faculty of Engineering, University of Porto, since 2018. Her investigation interests consist of the development of new materials for application in medical devices, specifically for tendon and ligament repair. 


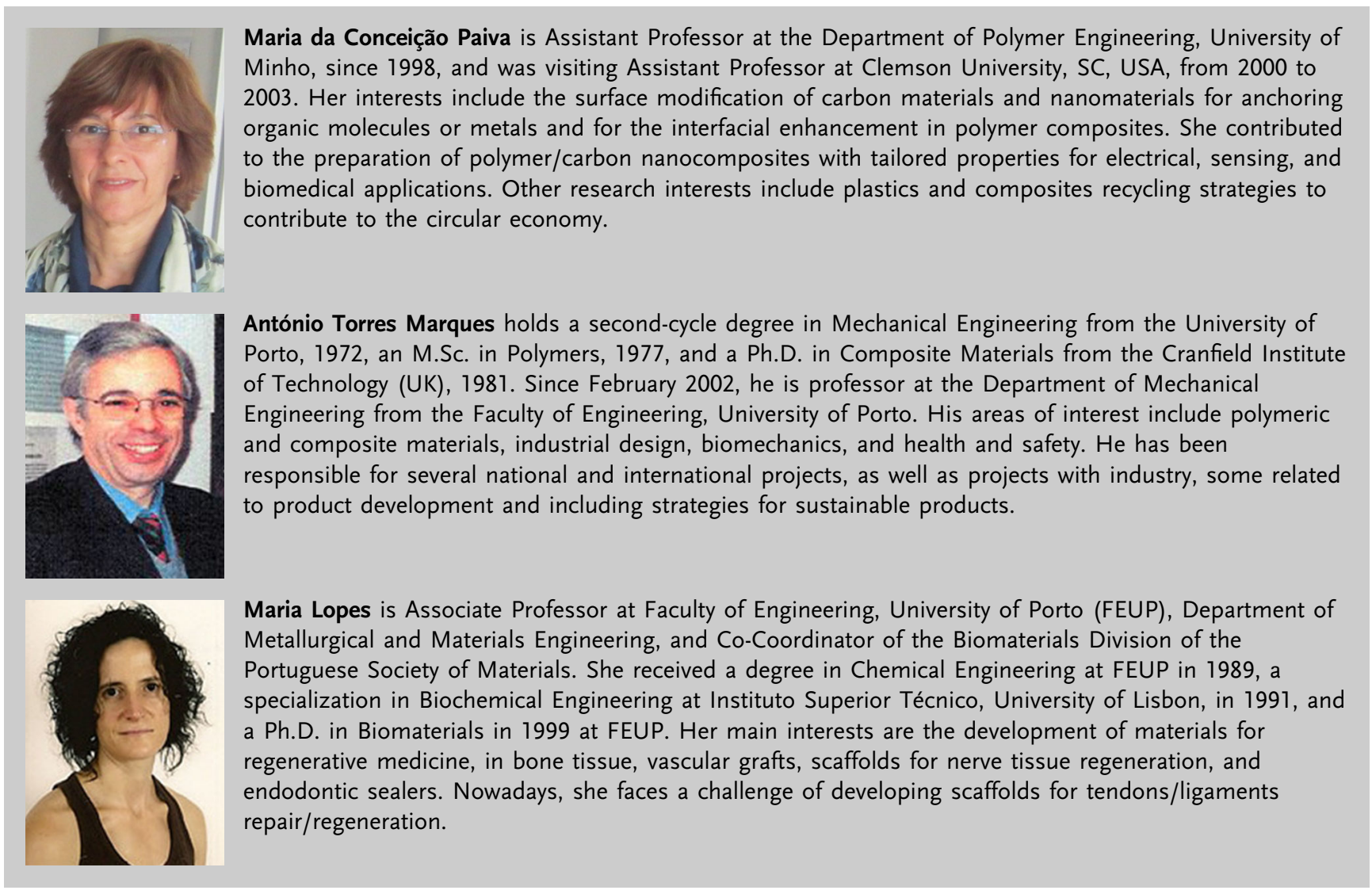

